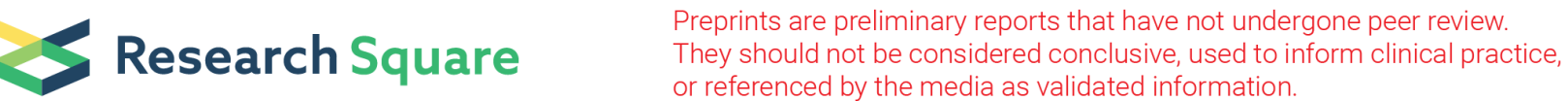

\section{Abnormal Lipid Droplets Accumulation Induced Cognitive Deficits in Obstructive Sleep Apnea Syndrome Mice via JNK/SREBP/ACC Pathway but Not Through PDP1/PDC Pathway}

\section{Dongze Li}

Binzhou Medical University

Yan Yu

Binzhou Medical University

$\mathrm{Na} \mathrm{Xu}$

Binzhou Medical University

Wanting Li

Binzhou Medical University

Yanyan Hou

Binzhou Medical University

\section{Xi Wang}

Binzhou Medical University

Yeying Sun

Binzhou Medical University

Wenxue Lu

Binzhou Medical University

Guiwu Qu

Binzhou Medical University

Changjun Lv

Binzhou Medical University

Fang Han ( $\sim$ Hanfangtuandui@163.com )

Binzhou Medical University

\section{Research}

Keywords: Chronic intermittent hypoxia, Cognitive deficits, ROS, Lipid droplets, JNK/SREBP/ACC pathway

Posted Date: October 5th, 2021

DOI: https://doi.org/10.21203/rs.3.rs-648813/v2 
License: (c) (i) This work is licensed under a Creative Commons Attribution 4.0 International License. Read Full License

Version of Record: A version of this preprint was published at Molecular Medicine on January 14th, 2022. See the published version at https://doi.org/10.1186/s10020-021-00427-8. 


\section{Abstract}

The mechanisms of chronic intermittent hypoxia $(\mathrm{ClH})$-induced cognitive deficits remain unclear. Here, our study found that 12 weeks $\mathrm{CIH}$ treatment induced lipid droplets (LDs) accumulation in hippocampal neurocytes of C57BL/6 mice, and caused severe neuro damage including neuron lesions, neuroblast (NB) apoptosis and abnormal glial activation. Studies have shown that the neuronal metabolism disorders might contribute to the $\mathrm{ClH}$ induced-hippocampal impairment. Mechanistically, the results showed that pyruvate dehydrogenase complex $\mathrm{E} 1 \otimes$ subunit (PDHA1) and the pyruvate dehydrogenase complex (PDC) activator pyruvate dehydrogenase phosphatase 1 (PDP1) did not noticeable change after intermittent hypoxia. Consistent with those results, the level of Acetyl-CoA in hippocampus did not significantly change after $\mathrm{ClH}$ exposure. Interestingly, we found that $\mathrm{CIH}$ produced large quantities of ROS, which activated the JNK/SREBP/ACC pathway in neurocytes. ACC catalyzed the carboxylation of Acetyl-CoA to malonyl-CoA and then more lipid acids were synthesized, which finally caused aberrant LDs accumulation. Therefore, the JNK/SREBP/ACC pathway played a crucial role in the cognitive deficits caused by LDs accumulation after $\mathrm{CIH}$ exposure. Additionally, LDs were peroxidized by the high level of ROS under $\mathrm{ClH}$ conditions. Together, lipid metabolic disorders contributed to neurocytes damage, which ultimately caused behavioral dysfunction. An active component of Salvia miltiorrhiza, SMND-309, dramatically alleviated these injuries and improved cognitive deficits of $\mathrm{ClH}$ mice.

\section{Introduction}

Chronic intermittent hypoxia $(\mathrm{ClH})$ is the main feature of obstructive sleep apnea syndrome (OSAS). In addition to hyperlipidemia, atherosclerosis, and high cardiovascular risk (Silvestrini et al,2002), ClH can also cause serious neurocognitive dysfunction, which is associated with regional alterations in hippocampus morphology (D'Rozario et al,2017). Currently, lipid droplets (LDs) accumulation has been referred to neurodegeneration (Liu et al,2017), such as hereditary spastic paraplegia (HSP) (Inloes et al,2018), Parkinson's disease (Han et al,2018) and Alzheimer's disease (Hamilton et al,2015).

Nevertheless, the role of LDs accumulation in $\mathrm{ClH}$-induced neuro dysfunction still needs to be determined. Under physiological conditions, the irreversible oxidative decarboxylation plays a central role in lipid homeostasis. Dephosphorylation of pyruvate dehydrogenase phosphatase 1 (PDP1) boosts the acetylation status of pyruvate dehydrogenase complex E1囚 subunit (PDHA1) and PDP1, which contributes to the subsequent pyruvate dehydrogenase complex (PDC) activation (Fan et al,2014). Then, activated PDC converts pyruvate to Acetyl-CoA (Walther et al,2017). As an important molecule in the metabolism processes of the human body, Acetyl-CoA is the raw material for de novo lipogenesis (Walther et al,2017). ACC, the rate-controlling enzyme in the pathway of lipogenesis, catalyzes the carboxylation of acetyl-CoA to malonyl-CoA (a highly regulated molecule in fatty acid synthesis) (Hunkeler et al,2018). As the production of $\mathrm{CIH}, \mathrm{ROS}$ is closely related to variety of physiological processes including lipid metabolism (Prabhakar et al,2012). Abnormal accumulation of ROS could regulate the expression of genes implicated in lipid metabolism, including SREBP, ACC, CPT-1, SCD-1 
(Zhao et al,2018). However, the potential mechanisms under abnormal lipid metabolism and its functional implication in diseases remains unknown.

SMND-309 is a major potent component extracted from salvia miltiorrhiza. It can be detected in the rat brain as a novel metabolite of salvianolic acid B after administration (Tian et al,2008). Research has proven that SMND-309 promotes neuron survival through antiapoptotic, anti-inflammatory and antioxidative effects (Yang et al,2010). In this study, SMND-309 was intraperitoneally injected into mice to alleviate $\mathrm{ClH}$-induced neuro damage. This might be helpful to develop a potential drug therapy target for OSAS.

\section{Material And Methods}

\subsection{Antibodies and reagents}

A rabbit anti-c-Jun N-terminal kinase (JNK) antibody (4668, CST), rabbit anti-phospho-JNK antibody (9252, CST), rabbit anti-Acetyl-CoA carboxylase (ACC) antibody $(3676$, CST), rabbit anti-NeuN antibody (24037, CST) and rabbit anti-cleaved caspase 3 (9664, CST), rabbit anti-PDP1 antibody (D8Y6L, CST) were purchased from Cell Signaling Technology (Massachusetts, USA). A rabbit anti-sterol regulatory element-binding protein (SREBP) antibody (ab28481, Abcam), rabbit anti-lba-1 antibody (ab178847, Abcam), goat anti-glial fibrillary acidic protein (GFAP) antibody (ab53554, Abcam), mouse antidoublecortin (DCX) antibody (ab135349, Abcam), rabbit anti- $\beta$-actin antibody (ab8227, Abcam), horseradish peroxidase-conjugated AffiniPure goat anti-rabbit IgG $(H+L)(a b 7090, A b c a m)$, Alexa Fluor 647 AffiniPure donkey anti-rabbit $\lg G(H+L)(a b 150063$, Abcam), Alexa Fluor 647 AffiniPure donkey antimouse IgG $(\mathrm{H}+\mathrm{L})$ (ab150111, Abcam), Alexa Fluor 594 AffiniPure donkey anti-rabbit lgG $(\mathrm{H}+\mathrm{L})$ (ab150064, Abcam), Alexa Fluor 594 AffiniPure donkey anti-goat IgG $(H+L)(a b 150136$, Abcam) and Alexa Fluor 555 AffiniPure donkey anti-rabbit IgG $(\mathrm{H}+\mathrm{L})(\mathrm{ab} 150066$, Abcam) were all obtained from Abcam (Cambridge, UK). A rabbit anti-p-PDHA1 antibody (ABS204m, Millipore) was from Millipore. Nile Red (72485) was purchased from Sigma-Aldrich. A rabbit anti-PDHA1 antibody (MA5-32545, Thermo), BODIPY (493/503) (D3922, Thermo Fisher) and C11-BODIPY (581/591) (D3861, Thermo Fisher) were from Thermo Fisher. SMND-309 (molecular formula $\mathrm{C}_{18} \mathrm{H}_{14} \mathrm{O}_{8}$, molecular weight 358.3) was provided by professor Guiwu Qu. The SMND-309 powder was dissolved in the normal saline, and the test mice were intraperitoneally injected at $25 \mathrm{mg} / \mathrm{kg}$ of body weight (Tian et al,2008)(Zhu et al,2013). 3-fluoropyruvate (3-FP, F4004, Sigma), a competitive inhibitor of PDHA1, was dissolved in PBS, and intraperitoneal injections of the 3-FP every 3 days at $80 \mathrm{mg} / \mathrm{kg}$ for 1 months (Chen et al,2018). In vitro experiment, the cells were added 3-FP to make the final concentration in the culture medium was $1 \mathrm{uM}$ after planted for 12 hours.

\subsection{Animals}

Six-week-old male wild-type (WT) mice on a C57BL/6 background (Jinan Pengyue Experimental Animal Breeding Co., Ltd., China) were used for this study. All mice were maintained at $23 \pm 1^{\circ} \mathrm{C}$ with a $12 \mathrm{~h} / 12 \mathrm{~h}$ light/dark cycle. Food and water were available ad libitum. Before the tests, 40 mice were randomly 
divided into 4 groups (WT + RA, WT + ClH, SMND-309, SMND-309 + ClH) / (WT + RA, WT + ClH, 3-FP, 3-FP + $\mathrm{ClH}$ ), and each group had 10 mice. Every mouse was given a unique number. During the whole testing process, the researchers were blinded to the treatment. All the mice were fed and used according to the $\mathrm{NIH}$ guidelines, and this study was approved by the Ethics Committees on Animal Experimentation of Binzhou Medical University (Permit no. SCXK20160006).

\subsection{Cell culture}

The neuron cell line HT22 (JNO-02001) was purchased from Guangzhou Jinio Biotechnology Co., Ltd. The cell was cultured with DMEM medium containing 10\% FBS, under standard incubation conditions at $37^{\circ} \mathrm{C}, 5 \% \mathrm{CO}_{2}$. Cell culture medium was replaced every 3 days and cells were passaged when they reached $80-90 \%$ confluency. The HT22 cells were seeded onto cover slips and randomly divided into four groups: normal control (NC) group ( $n=4$; RA exposure), intermittent hypoxia $(\mathrm{IH})$ group $(\mathrm{n}=4$; $\mathrm{IH}$ exposure), NC + 3-FP group ( $n=4$; RA exposure and 3-FP treatment), and $\mathrm{IH}+3-\mathrm{FP}$ group $(\mathrm{n}=4 ; \mathrm{IH}$ exposure and 3-FP treatment).

\subsection{Low oxygen exposure 2.4.1. ClH exposure}

The mice were exposed to $\mathrm{ClH}$ /room air (RA) conditions from 8:00 AM-4:00 PM per day for 12 weeks. During the exposure, the mice in the WT + CIH group and the SMND-309 + CIH (3-FP + CIH) group were placed into the CIH chamber (BioSpherix OxyClycler A84, USA), the WT + RA group and the SMND-309 (3FP) group were placed into the RA chamber (BioSpherix OxyClycler A84, USA). The CIH program was performed as described previously (Li et al,2020). Firstly, the chamber was filled with $\mathrm{N}_{2}$ for $85-95 \mathrm{~s}$, and the oxygen level was reduced from $21 \% \pm 1 \%$ (normal) to $7 \% \pm 1 \%$ (hypoxia). The oxygen level was then maintained at $7 \% \pm 1 \%$ for $15-20$ s. Finally, the level was recovered to $21 \% \pm 1 \%$ in $45-50$ s and sustained for 15-20 s. A cycle of $\mathrm{ClH}$ lasted approximately $180 \mathrm{~s}$, and there were approximately 20 cycles per hour. The oxygen level in the RA chamber was always maintained at $21 \% \pm 1 \%$.

\subsubsection{IH exposure}

$\mathrm{IH}$ was induced by culturing cells in an oxygen control cabinet (Biospherix Oxycycler C42) mounted within an incubator and equipped with oxygen sensor for continuous oxygen level monitoring. A mixture of nitrogen oxygen and $5 \% \mathrm{CO}_{2}$ was infused and oxygen levels consisted of a 30 -minute hypoxic period (3\% O2), followed by 30 minutes of reoxygenation $\left(21 \% \mathrm{O}_{2}\right)$. Actual $\mathrm{O}_{2}$ saturation was kept at $3 \%$ for 10 minutes, at each 1-hour cycle (Dyugovskaya et al,2012). This IH treatment was performed for 3 day.

\subsection{Behavioral testing}

\subsubsection{Three-chamber social test}

To examine cognitive function impairment due to $\mathrm{ClH}$ treatment, the three-chamber social test, Morris water maze (MWM) test and fear conditioning test were performed. The three-chamber social test was 
conducted as previously described (Moy et al,2004). The chamber used in the test $(60 \mathrm{~cm} \mathrm{~L} \times 26 \mathrm{~cm} \mathrm{~W} \times$ $30 \mathrm{~cm} \mathrm{H}$ ) was divided into three sections: center, left and right $(20 \mathrm{~cm} \mathrm{~L} \times 26 \mathrm{~cm} \mathrm{~W})$. The test mouse had free access to the different sides of the chamber through the door $(11 \mathrm{~cm} \mathrm{~L} \times 11 \mathrm{~cm} \mathrm{~W})$ on the dividing wall. The test consisted of 3 phases with 20 min intervals. Each phase allowed the test mouse to explore the chamber for $10 \mathrm{~min}$. During the phase, the video system tracked the behavior of the mouse. At phase 1 , a test mouse was placed in the center chamber, and two empty wire cages $(10 \mathrm{~cm} \mathrm{D} \times 14 \mathrm{~cm} \mathrm{H})$ were placed into the left and right sides. During phase 2, an unfamiliar (stranger) mouse was placed in the left wire cage, and an empty wire cage was placed on the right side. Another stranger mouse was then placed in the right wire cage in phase 3. Mice were tested from approximately 8:00 AM to 12:00 AM and received $\mathrm{ClH} / \mathrm{RA}$ treatment from 12:00 AM to 4:30 PM.

\subsubsection{MWM}

The MWM test was conducted as previously described (Lee et al,2019). The system contained a circular pool $(122 \mathrm{~cm} \mathrm{D} \times 51 \mathrm{~cm} \mathrm{H})$ and an escape platform $(12 \mathrm{~cm} \mathrm{D} \times 34 \mathrm{~cm} \mathrm{H})$ (ZS Dichuang, Beijing, China). The pool was divided into four equal quadrants and filled with $35 \mathrm{~cm}$ of deep water. The water was dyed with $\mathrm{TiO}_{2}$ and kept at $23 \pm 1{ }^{\circ} \mathrm{C}$. The escape platform was positioned $1 \mathrm{~cm}$ below the water surface in quadrant two. The same discriminate landmarks were placed around the maze during the test. The test consisted of two stages, including four days of the training stage and one day of the probe trial stage.

The day before the test, all the mice were acclimated to the pool without the platform for $60 \mathrm{~s}$. During the training stage, the test mouse performed four trials for 4 consecutive days. In the first trial, a mouse was put into the water facing the pool wall at quadrant one. If the mouse found the escape platform in $60 \mathrm{~s}$, the tracker system would be automatically closed; otherwise, the mouse would be guided to the platform and forced to stay on it for $15 \mathrm{~s}$. With a 15 min interval, the mouse received a second trial and started at quadrant two. After trials 3 and 4, the mouse was dried with a towel and placed back into the home cage. At the probe trial stage (day 5), the mouse was put into the water at quadrant four and forced to swim without the platform for $60 \mathrm{~s}$. Several performance parameters of the test mouse were recorded, including the total swimming distance, the number of platform crossings, the duration and the distance traveled in the target quadrant. The tests were performed from 8:00 AM to 12:00 AM. After the tests, the mice were exposed to $\mathrm{ClH} / \mathrm{RA}$ conditions from 12:00 AM to 4:30 PM.

\subsubsection{Fear conditioning test}

The equipment consisted of a conditioning chamber with a sound-attenuating wall and a video monitoring system (ZS Dichuang, Beijing, China). An acrylic box ( $35 \mathrm{~cm} \mathrm{~L} \times 35 \mathrm{~cm} \mathrm{~W} \times 35 \mathrm{~cm} \mathrm{H}$ ) and a stainless-steel grid floor were placed in the chamber. The floor was connected to a device to deliver the footshock, and tests were performed as previously described (Shoji et al,2014). On day 1, after acclimating the chamber (black acrylic box with a jasmine smell) for 2 min, the test mouse underwent 30 $\mathrm{s}$ of sound $(65 \mathrm{~dB}, 3 \mathrm{kHz})$ three times (separated by 30 -second intervals), and the mouse received a foot shock $(0.7 \mathrm{~mA})$ during the last $2 \mathrm{~s}$ of each sound. On day 2 , the mice were exposed to the same environment (black acrylic box with a jasmine smell) for 5 min with no sound and no foot shock. The fear 
behavior (freezing) of the mouse, which referred to contextual memory, was recorded. On day 3 , after 2 min of acclimatization to the new environment (blue acrylic box with a lemon smell), the mouse was exposed to $30 \mathrm{~s}$ of sound ( $65 \mathrm{~dB}, 3 \mathrm{kHz}$ ) three times (separated by 30 -second intervals). During this stage, the fear behavior (freezing), which refers to cued memory, was measured.

\subsection{Tissue preparation}

Five mice from each group were deeply anesthetized by $4 \%$ choral hydrate through intraperitoneal injection. They were then perfused with physiological saline via the left ventricle of the heart, and fixed with $4 \%$ ice-cold paraformaldehyde. The fixed brains were embedded in optimal cutting temperature compound, frozen in liquid nitrogen and stored at $-80^{\circ} \mathrm{C}$. For the other mice, the hippocampi were harvested and stored in liquid nitrogen.

\subsection{Transmission electron microscopy (TEM)}

The hippocampi of each group were fixed in 2.5\% ice-cold glutaraldehyde in $0.1 \mathrm{M} \mathrm{PBS}$ at pH 7.4 and osmium tetroxide. After gradient dehydration with ethyl alcohol and acetone, the hippocampi were embedded in epoxy resin Epon 812. Tissues were then cut into ultrathin sections ( $50 \mathrm{~nm}$ ) by a CM1900 microtome (Leica, Germany) and stained with uranyl acetate and lead citrate. Samples were viewed in a JEOL JEM 1010 TEM at $80 \mathrm{kV}$ and captured through an AMT XR-16 mid-mount 16 mega-pixel digital camera (Hu et al,2011).

\subsection{Hematoxylin and eosin (H\&E) staining}

The brains of each group were cut into $5 \mu \mathrm{m}$ sections and stained with H\&E. After dehydration with ethanol, the sections were observed by an Invitrogen EVOS M5000 imaging system (Thermo Fisher, USA) (Ballok et al,2004).

\subsection{Nile red staining}

To detected the accumulation of LDs, the frozen slices were washed three times in PBS and incubated with Nile red (1:1000) for 10 min. After washing three times with PBS, the slices were mounted with Vectashield with DAPI (Vector Labs, USA) and imaged with an Invitrogen EVOS M5000 imaging system (Thermo Fisher, USA). The Nile red powder was dissolved in methyl alcohol at a concentration of 1 $\mathrm{mg} / \mathrm{mL}$.

\subsection{Measure the levels of PDC activity, Acetyl-CoA and ROS}

Pyruvate Dehydrogenase Activity Assay Kit (MAK183) was purchased from Sigma. Acetyl-Coenzyme A Assay Kit (Sigma, MAK183) was used to determine the Acetyl-CoA level. The contents of ROS in the hippocampus were detected with an Aconitase Activity Assay Kit (Sigma-Aldrich, MAK051) (Liu et al,2015). All the protocols were conducted in accordance with the manufacturer's instructions. The optical density of the wells was detected by the Ultra Multitask Ascent (Epoch, BioTek, USA). 


\subsection{Immunofluorescence}

After washing three times with PBS, the frozen slices were permeabilized with $0.3 \%$ Triton X-100 (T8200, Solarbio, Beijing, China) in PBS for 5 min. The slices were then incubated with $10 \%$ donkey serum (Jackson ImmunoResearch, Pennsylvania, USA) for $30 \mathrm{~min}$ at room temperature and were then incubated with primary antibodies including mouse anti-DCX (1:200), rabbit anti-cleaved-caspase 3 (1:200), rabbit anti-Iba-1 (1:200), goat anti-GFAP (1:300) or rabbit anti-NeuN (1:200) overnight at $4^{\circ} \mathrm{C}$. The secondary antibodies were maintained at room temperature for $1 \mathrm{~h}$ and included Alexa Fluor 647 AffiniPure donkey anti-rabbit (1:500), Alexa Fluor 647 AffiniPure donkey anti-mouse (1:500), Alexa Fluor 594 AffiniPure donkey anti-rabbit (1:500), Alexa Fluor 594 AffiniPure donkey anti-goat (1:500) or Alexa Fluor 555 AffiniPure donkey anti-rabbit (1:500). After staining with BODIPY (493/503) (1:200) for 15 min or C11BODIPY (581/591) (1:500) for 30 min and washing the sections three times in PBS, the sections were mounted with Vectashield with DAPI (Vector Labs, USA) and imaged using an Invitrogen EVOS M5000 imaging system (Thermo Fisher, USA) [23]. The BODIPY (493/503) powder was dissolved in PBS at 1 $\mathrm{mg} / \mathrm{mL}$, and the C11-BODIPY (581/591) powder was dissolved in DMSO at $2 \mathrm{mg} / \mathrm{mL}$.

\subsection{Protein extraction and western blot}

After suspending the proteins in RIPA lysis buffer (R0020, Solarbio, Beijing, China), the protein concentrations were measured by a bicinchoninic acid assay kit (PC0020, Solarbio, Beijing, China). The samples were then homogenized in SDS loading buffer (9173, TaKaRa, Beijing, China) and separated through SDS-PAGE with 8-12\% gels (P0012, Beyotime, Shanghai, China). After being transferred to polyvinylidene difluoride membranes (03010040001, Roche, USA) in an ice bath, the membranes were blocked with $5 \%$ nonfat dry milk in TBST at room temperature. They were then incubated with an antiJNK antibody (1:1000), anti-P-JNK antibody (1:1000), anti-SREBP antibody (1:1000), anti-ACC antibody (1:1000), anti-PDP1 antibody (1:1000), anti-PDHA1 antibody (1:5000), anti-p-PDHA1 antibody (1:2000) or anti- $\beta$-actin antibody $(1: 5000)$ overnight at $4^{\circ} \mathrm{C}$. After washing three times with TBST, the membranes were incubated with the horseradish peroxidase-conjugated secondary antibody (1:5000) at room temperature for $1 \mathrm{~h}$. Finally, the blots were developed using an electrochemiluminescence detection system (ChemScope 6000, Clinx Science Instruments CO., Ltd., China).

\subsection{RT-PCR}

The total RNA was prepared from hippocampal tissue samples or cells using Trizol reagent (Invitrogene) following the manufacturer's instructions. Isolated total RNA was quantified spectrophotometrically. Aliquots of total RNA were reverse transcribed using cDNA synthesis kit (Takara RR047A) according to the manufacturer's protocol. PCR primers (PDHA1, FP-5'-GAAATGTGACCTTCATCGGCT-3', RP-5'TGATCCGCCTTTAGCTCCATC-3'; PDP1, FP-5'-GCACCCATAGAGGACCGGA-3', RP-5'CCTGCATGACCATCAAAAACCC-3') were purchased from Thermo Fisher. Standard curves for gene of interest and housekeeping gene ( $\beta$-actin) were included in each reaction. Using SYBR Green PCR Master Mix to quantify, 40 cycles of RT-PCR were performed on an ABI 7500 thermal cycler (Applied Biosystems). 


\subsection{Statistical analysis}

All experiments were repeated three times. The experimenter was unaware of the animal's group during experimentation and removed the mice that were in poor condition (they did not like to exercise). SPSS version 24.0 (IBM Crop, Chicago, USA) was used for statistical analysis. Data are displayed as the mean \pm SEM. One-way analysis of variance (ANOVA) and a Bonferroni post hoc test were used to evaluate the results of the three-chamber social tests (Xu et al,2019). The results of escape latency in the MWM test were examined through three-way mixed ANOVA (Champagne et al,2002). For the total traveling distance, platform crossing counts, the duration and distance in the target zone and the fear conditioning tests were assessed with two-way ANOVA (Van Dam et al,2000). Other data were assessed with two-way ANOVA. Statistical significance was accepted at $p<0.05$.

\section{Results}

\subsection{The exposure of $\mathrm{ClH}$}

The mice were exposed to RA/ClH condition for 12 weeks (shown in Fig. 1. B). During the last week of the exposure, the mice were intraperitoneal injected with SMND-309 for once daily at $25 \mathrm{mg} / \mathrm{kg}$ of body weight. The three-chamber social test, the MWM test and the fear conditioning test were conducted in sequence as soon as the treatment was finished (shown in Fig. 1. A).

\section{2. $\mathrm{CIH}$ exposure caused the social damage, SMND-309 could attenuate the damage}

At phase 1, there was no obvious side preference in the 4 groups (shown in Fig. 2. A, D). During phase 2, all the mice preferred to stay with the stranger mouse than at the inanimate side $(p<0.05$, shown in Fig. 2. B, E). At phase 3, WT + RA mice $\left(F_{(2,27)}=55.839, T=6.827, p<0.05\right)$ and SMND-309 mice $\left(F_{(2,27)}=\right.$ $71.527, T=6717, p<0.05$ ) preferred to stay with the new stranger mouse than the familiar one (shown in Fig. 2. C). However, $\mathrm{WT}+\mathrm{ClH}$ mice spent similar time at the two sides $\left(F_{(2,27)}=18.716, p>0.05\right)$ (shown in Fig. 2. C) during this stage. Treated with SMND-309, SMND-309 + ClH mice spend increasing time with the stranger mouse than the familiar one $\left(F_{(2,27)}=37.689, T=3.718, p<0.05\right)$ (shown in Fig. 2. C). The traveling distance of phase 3 showed same pattern with spending time for all groups (shown in Fig. 2. F). These results showed that $\mathrm{ClH}$ treatment damaged the social novelty cognition of mice and SMND-309 could alleviate the damage.

\section{3. $\mathrm{ClH}$ exposure induced spatial learning and memory deficits, SMND-309 could relieve}

During the training days, the mean escape latency of WT + RA and SMND-309 mice decreased rapidly, but it had almost no change in the WT $+\mathrm{CIH}$ group (day 4: $F_{(3,156)}=5.626, T=3.585, p<0.05$ ) (shown in 
Fig. 2. G). At the probe trial stage, the total swimming distance of all groups was similar $\left(F_{(3,36)}=1.564, p\right.$ $=0.215$ ) (shown in Fig. $2 . \mathrm{H}$ ). In contrast, the $\mathrm{WT}+\mathrm{ClH}$ group showed a remarkable reduction in the number of platform crossings $\left(F_{(3,36)}=3.28, T=3.725, p<0.05\right)$ (shown in Fig. 2. I). The duration $\left(F_{(3,36)}\right.$ $=7.266, T=3.939, p<0.05)$ (shown in Fig. $2 . J$ ) and the distance $\left(F_{(3,36)}=4.007, T=3.963, p<0.05\right.$ ) (shown in Fig. 2. K) traveled at the target quadrant were also shorter for the $\mathrm{WT}+\mathrm{ClH}$ group than for the WT + RA group. After SMND-309 treatment, these reductions were notably reversed. These data indicated that $\mathrm{ClH}$ exposure impair the spatial learning and memory of mice, but SMND-309 relieved the damage.

\subsection{CIH exposure induced fear memory impairment, SMND- 309 could improve}

Twenty-four hours after the footshock, WT $+\mathrm{ClH}$ mice presented with a lower level of freezing than WT + RA mice $\left(F_{(3,36)}=14.139, T=6.82, p<0.05\right)$, but this change was improved after treatment with SMND309 in SMND-309 + ClH group $\left(F_{(3,36)}=14.139, T=4.079, p<0.05\right)$ (shown in Fig. 2. L). Similar patterns could also be observed in the cued memory test (day 3) (shown in Fig. 2. M). In conclusion, the fear memory of mice was severely injured under $\mathrm{ClH}$ condition, and this injure could be ameliorated by SMND309 treatment.

\subsection{ClH-induced pathological changes in the hippocampus}

After $\mathrm{ClH}$ treatment, severe histological changes have been revealed, neurons are loosely arranged, irregular sized, with fuzzy outline. Meanwhile, the number of neuroblasts (NBs) in the hippocampal subgranular zone (SGZ) was significantly decreased (shown in Fig. 3. A). This injury was relieved by SMND-309 treatment (shown in Fig. 3. A). As seen with TEM, the neuron ultrastructure was completed, and the blood-brain barrier (BBB) was thin and intact in the WT hippocampus (shown in Fig. 3. B). However, neurons exhibited cytoplasmic vacuolization and mitochondrial disintegration under $\mathrm{ClH}$ conditions. Moreover, a large number of LDs appeared in the damaged neurons (shown in Fig. 3. B). For astrocytes, severe edema could be observed, especially in the area surrounding the BBB (shown in Fig. 3. B).

\subsection{LDs-induced NBs apoptosis after CIH exposure, SMND- 309 could ameliorate}

The fluorescence of Nile red was dim in the dentate gyrus (DG) area of WT + RA mice and SMND-309 mice (shown in Fig. 4. A), but the number of LDs increased 2-fold after $\mathrm{ClH}$ treatment $(p<0.05)$. Interestingly, it decreased 45\% after SMND-309 treating in SMND-309 + CIH group $(p<0.05)$ (shown in Fig. 4. A). To confirm the distribution of LDs in different types of neurocytes, cell-type markers NeuN, GFAP, Iba- 1 and LDs dye BODIPY (483/503) were used. Following $\mathrm{CIH}$ treatment, the fluorescence spots of BODIPY $(483 / 503)$ enhanced 8-fold in the neurons $(p<0.05)$ (shown in Fig. 4. B), nearly 3-fold in the astrocytes $(p<0.05)$ (shown in Fig. 5 ) and 1 -fold in the microglia $(p<0.05)$ (shown in Fig. 5$)$ of the DG area than WT + RA group. Intriguingly, SMND-309 noticeably decreased 70\% of LDs accumulation in the 
neurons $(p<0.05)$ (shown in Fig. 4. B), 40\% in the astrocytes $(p<0.05)$ (shown in Fig. 5$)$ and $30 \%$ in the microglia $(p<0.05)$ (shown in Fig. 5).

To determine the damage of NBs in the SGZ by LDs accumulation, the NB marker DCX and the apoptosis marker cleave-caspase 3 was stained with the LDs dye BODIPY (483/503). The number of DCX-positive cells in the DG area was decreased $80 \%$ after $\mathrm{CIH}$ treatment $(\mathrm{p}<0.05)$ (shown in Fig. 6). In addition, the quantity for cleaved caspase 3-positive cell increased $85 \%$ with BODIPY $(483 / 503)$ spots in the DG area of $\mathrm{WT}+\mathrm{CIH}$ mice $(\mathrm{p}<0.05)$ (shown in Fig. 6). Notably, apoptosis NB significantly reduced in SMND-309+ $\mathrm{ClH}$ group than WT $+\mathrm{ClH}$ group $(p<0.05)$ (shown in Fig. 6).

\subsection{Affecting the pathway of PDP1/PDHA1 did not improve the damage caused by $\mathrm{ClH}$ exposure}

The mice were exposed to $\mathrm{RA} / \mathrm{ClH}$ condition for 12 weeks, and received intraperitoneal injection with 3-FP every 3 days at the last 3 weeks of the exposure(shown in Fig. 7. A). The fear conditioning test were conducted as soon as the treatment was finished (shown in Fig. 7. A).

In the context memory test, WT $+\mathrm{ClH}$ mice presented with a lower level of freezing compared with WT + RA group than mice. It should be noted that this change was not improved after treatment with PDHA1 inhibitor 3-FP (shown in Fig. 7. C, ${ }^{*} p<0.05$ ). After $48 \mathrm{~h}$ retention delay, the cued fear conditioning testing were determined, the freezing level was similar to the trend of context memory test (shown in Fig. 7. D, *p $<0.05$ ). Taken together, these results provided evidence that $\mathrm{ClH}$ exposure contributed to the long-term memory dysfunction, and PDHA1 inhibitor 3-FP could not improve the damage.

The H\&E stained of hippocampus section in 4 groups were shown in Fig. 7E. Compared to the RA group, severe histological changes had been revealed in $\mathrm{ClH}$ group. Hippocampal granular neurons were loosely arranged,some neurons had showed a swollen and vacuolated cytoplasm and the neural progenitor cells in the DG area were significantly reduced. Significantly, this injury was not relieved by PDHA1 inhibitor treatment.

\subsection{PDP1/PDHA1 did not significantly promote the increase of LDs induced by $\mathrm{ClH}$ in hippocampus}

The fluorescence of Nile red showed that the $\mathrm{ClH}$ group and $\mathrm{ClH}+3-\mathrm{FP}$ group had sporadic superficial, round or elongated lipid-containing structures in the hippocampus, indicating abnormal accumulation of LDs (shown in Fig. 8. A, B). Real time RT-PCR analysis and Western blot analysis were performed to analyze the expression level of PDP1 and PDHA1. Quantitatively, the gene expression and protein levels of PDP1 and PDHA1 showed no significant differences among 4 groups (shown in Fig. 8. C-G). The protein level of phosphorylated PDHA1 (p-PDHA1) (shown in Fig. 8. H), the activity of PDC (shown in Fig. 8. I) and the content of Acetyl-CoA (shown in Fig. 8. J) were tested to further explore the role of PDP1/PDHA1 related lipid synthesis on $\mathrm{ClH}$ condition. All these results showed no statistically differences between groups, which suggested $\mathrm{ClH}$ exposure almost had no impact on the Acetyl-CoA 
biosynthesis regulated by PDP1/PDHA1. In vitro experiments supported this conclusion (shown in Fig. Sp. 1).

Experimental data showed that the PDP1/PDHA1 may not be the main source of the abnormal increased lipids after $\mathrm{ClH}$ exposure. Therefore, other reasonable mechanisms of lipids synthesis disorder in neurocytes under stress needed to be interpreted.

\subsection{ROS-triggered JNK/SREBP/ACC pathway activation and lipid peroxidation, SMND-309 could decrease}

To confirm the level of ROS in the hippocampus under $\mathrm{ClH}$ conditions, an aconitase activity assay kit was used. The results showed that the activity for aconitase decreased $50 \%$ in WT $+\mathrm{ClH}$ group $(\mathrm{p}<0.05)$ (shown in Fig. 9. G). Furthermore, the expression of phosphorylated JNK increased nearly 1.5-fold $(p<$ 0.05 ), and this effect activated SREBP and upregulated the expression of ACC for 3 -fold $(p<0.05)$ (shown in Fig. 9. A-F). Treated with SMND-309 eliminated ROS $(p<0.05)$ (shown in Fig. 9. G) and inhibited the activation of the JNK/SREBP/ACC pathway (shown in Fig. 9. A-F).

ROS are oxidation products and cause damage to lipids. Peroxidized lipids could be observed after staining with the lipid peroxidation dye C11-BODIOY (581/591), results indicated that the number of peroxidated lipid increased 2-fold in the hippocampal neurocytes of WT $+\mathrm{ClH}$ mice $(p<0.05)$ (shown in Fig. 10). Notably, it reduced nearly $50 \%$ after SMND-309 treatment $(p<0.05)$.

\section{Discussion}

As a common sleep disorder disease, OSAS is characterized by sudden pauses of breathing during sleep ( $\mathrm{Li}$ et al,2018). Resulting from repeated obstructions of the pharyngeal airway, $\mathrm{CIH}$ is a cardinal feature of OSAS, which induces degrease of cognitive performance. Our research found that the ability of social memory and spatial memory was damaged in $\mathrm{C} 57 \mathrm{BL} / 6$ mouse after $\mathrm{ClH}$ treatment. Further study discovered that the hippocampal neurocytes of those mice were severely injured. It is thought that hippocampus is crucial for encoding new memory (Suh et al,2011). Therefore, the injury of hippocampus by $\mathrm{CIH}$ finally gave rise to behavioral deficits. Until now, researchers consider that the mechanism of those neuro damage is related to the inflammation and the oxidative stress. In response to $\mathrm{CIH}$, immune receptors initiate chronic neuroinflammation, such as TLR 2 and TLR 4. Those receptors upregulate the expression of inflammation cytokines, including IL-1 $\beta$, IL-6 and TNF- $\alpha$ via TLR 2/TLR 4-MyD88 signal pathway ( $\mathrm{Li}$ et al,2020)(Lu et al,2019). In this process, microRNA (Ren et al,2019), histone modifications and DNA methylation (Kiernan et al,2016) have also been involved in. Besides, hypoxia-reoxygenation injures cell organs by promoting ROS generation. Targeting mitochondrial protein OPA1, ROS induces mitochondrial fission and disturbs mitochondrial membrane potential (Rovira-Llopis et al,2017). Also, ROS initials apoptosis through triggering ER stress and ER calcium release (Ding et al,2016).

Recently, LDs accumulation is closely related to a variety of human diseases (Maxfield \& Tabas,2005). In peripheral system, LDs-associated protein cell death-inducing DFF45-like effector (CIDE), which is crucial 
for the formation and fusion of LDs, regulates the occurrence of type Il diabetes (Dahlman et al,2005). The depletion of perilipin 2 prevents hepatic steatosis via downregulating triglyceride synthesis and LDs accumulation (Carr et al,2014). In nervous system, LDs accumulation enhances the formation of oligomeric a-synuclein, a major component of the pathological hallmarks in Parkinson's disease (Ruipérez et al,2010). Knocking out HSP-related genes leads to LDs accumulation in neurons (Klemm et al,2013). LDs accumulation also disrupts energy homeostasis (Konige et al,2014), impairs the folding and clearance of proteins in neurons (Inloes et al,2018), and breaks neuron-glia metabolic coupling (Schmitt et al,2014). All these studies have shown that LDs accumulation is closely related to neurodegeneration diseases. Contradictorily, some studies support that LDs accumulation is helpful for neural protection. In the glial niche of Drosophila larvae, LDs accumulation keeps neural stem cells away from oxidative damage (Moldavski et al,2015). Some fatty acids are vulnerable to peroxidation, and they are diverted into LDs to protect from ROS under hypoxia condition (Welte \& Gould,2017). Therefore, exploring the molecular mechanisms of altered lipid metabolism in brain injury, will help to reveal the cause of neurodegenerative changes, including $\mathrm{ClH}$-induced cognitive dysfunction.

PDP1/PDHA1 pathway is an essential regulatory for de novo lipid synthesis. PDHA1 is activated by dephosphorylation of PDP1 and phosphorylation of pyruvate dehydrogenase kinases (PDK) (Shan et al,2014). PDHA1 is one of the most important components of the PDC (Zhong et al,2017), which oxidates pyruvate to Acetyl-CoA. As a major and central precursor in metabolism, Acetyl-CoA candidates in the synthesis and decomposition of biomacromolecules, especially for lipid biosynthesis (Kuerschner et al,2008). It has been reported that PDP1/PDHA1 was related to a variety of diseases by affecting lipid metabolism. Inactivation of PDHA1 suppresses tumourigenesis by decreasing Acetyl-CoA levels in prostate cancer. However, knocking out PDK4 alleviates the hepatic steatosis by regulating the activity of PDC in nonalcoholic steatohepatitis mouse models (Zhang et al,2018). In this study, experimental data showed that the activity of PDC and the production of Acetyl-CoA did not noticeable change after $\mathrm{ClH}$ exposure, which suggested the de novo lipid synthesis regulated by PDP1/PDHA1 might not be the main source of the abnormal increased lipids and LDs after $\mathrm{ClH}$ exposure. Therefore, the relevant mechanism still needs to be further studied.

ROS production and oxidative stress participate in neuro injuries and neurodegeneration. In Parkinson disease, ROS induces missense mutation by damaging DNA and inevitably causing neural cell damage (Pignataro et al,2017). ROS also evokes Alzheimer's disease through active NLRP3, which promotes IL-1 $\beta$ mediated inflammation (Pignataro et al,2017). Recently, studies found that ROS is capable to promote lipid synthesis and LDs accumulation (Liu et al,2015). Increased level of ROS exerts harmful effects by causing oxidative damage to biological macromolecules and disrupting various signaling pathways including the lipid metabolism (Fransen et al,2012). In the development of fatty liver, redox cellular state especially the high level of ROS activates lipid biosynthesis gene SREBP and speeds up the disease process (Pan et al,2017). Reports show that ROS triggers SREBP activity in fruit fly neurons leading to LDs accumulation (Liu et al,2015). SREBP regulates the expression of several genes, such as ACC and fatty acid synthase (FAS), which is the candidate of cellular fatty acid synthesis (Nogalska et al,2005). ACC is a rate-limiting enzyme in de novo fatty acid synthesis, catalyzing ATP-dependent carboxylation of 
Acetyl-CoA to form malonyl-CoA (an intermediate in fatty acid biosynthesis) (Hunkeler et al,2018). We found that high level of ROS triggered JNK/SREBP/ACC pathway activated in neurons, thus more AcetylCoA were converted into fatty acid after $\mathrm{ClH}$ exposure. The excessive increase of lipid synthesis promoted abnormal LDs accumulation, which severely injured neurocytes in hippocampus. In addition, lipid peroxidation further aggravated neuro damage.

SMND-309 is a degradation production of Salvia miltiorrhiza, which has been widely used for neuroprotection (Su et al,2015). SMND-309 inhibits apoptosis by upregulating the ratio of Bcl-2/Bax (Yang et al,2010) and promotes neuron survival by increasing the content of brain-derived neurotrophic factor via activating the phosphatidylinositol 3-kinase/Akt/cAMP-response element-binding (CREB) signaling pathway (Wang et al,2016). Consistent with the results of previous studies, SMND-309 treatment improved the behavioral performance of $\mathrm{CIH}$ mice by reducing the accumulation of LDs in neurocytes of the DG area. These findings might be helpful to provide a novel potential neuroprotective therapy.

In this study, $\mathrm{ClH}$-induced hippocampal damaging was triggering by LDs accumulation in NBs, neurons and glia cells. The generation of LDs could be regulated via JNK/SREBP/ACC pathway. And these damages were alleviated by SMND-309 treatment. Until now, the role of LDs in neurocyte is controversial. The mechanism of lipid synthesis disorder and LDs abnormal accumulation is also unclear. All these questions need to be further investigated.

\section{Conclusion}

Present study found that cognitive function was severely damaged in $\mathrm{ClH}$ mice because of neurocyte injuries in the hippocampal DG area. After CIH exposure, the expression of PDP1/PDHA1, the activity of PDC and the level of cellular Acetyl-CoA have barely changed. Noticeably, ROS triggered JNK/SREBP/ACC pathway, and then more Acetyl-CoA were converted into fat acids. The redundant lipids led to aberrant LDs accumulation, which contributed to the neuro injuries. What's more, lipid peroxidation as a result of excessive ROS also promoted the damage in neurocytes. Remarkably, all of these damages induced by lipid metabolic disorders were relieved by SMND-309 treatment.

\section{Abbreviations}

$\mathrm{ClH}$, chronic intermittent hypoxia; IH, intermittent hypoxia; LDs, lipid droplets; NBs, neuroblasts; JNK, cJun-N-terminal Kinase; SREBP, sterol regulatory element binding protein; ACC, Acetyl CoA carboxylase; OSAS, obstructive sleep apnea syndrome; SGZ, subgranular zone; HSP, hereditary spastic paraplegia; PDP1, pyruvate dehydrogenase phosphatase 1; PDHA1, pyruvate dehydrogenase complex E1囚 subunit; PDC, pyruvate dehydrogenase complex; GFAP, glial fibrillary acidic protein; DCX, doublecortin; WT, wild type; RA, room air; NC, normal control; 3-FP, 3-fluoropyruvate; MWM, Morris water maze; TEM, transmission electron microscopy; H\&E, Hematoxylin and eosin; ANOVA, analysis of variance; BBB, blood- 
brain barrier; CIDE, cell death-inducing DFF45-like effector; PDK, pyruvate dehydrogenase kinases; PSANCAM, polysialylated form of the neural cell adhesion molecule; CREB, cAMP-response element-binding.

\section{Declarations}

\section{Acknowledgements}

Not applicable.

\section{Authors' contributions}

YY, CJL and FH designed this study and the structure of the paper, GWQ provided drugs. DZL, NX, and YYH performed experiment and analyzed results. WJR and NZ collected the information. DZL, XW, YYS, and WXL wrote the manuscript draft. YY, DZL and NZ helped to submit the manuscript. All authors read and approved the final manuscript.

\section{Funding}

This project was funded by National Natural Science Foundation of China (No.81600069), the Scientific Research Foundation of collaborative innovation (the prevention and treatment of sleep and respiratory related diseases), and the Scientific Research Foundation of Fang Han Team.

\section{Availability of data and materials}

The data that support the findings of this study are available from the corresponding author upon reasonable request.

\section{Declarations}

\section{Ethics approval and consent to participate}

All the mice were fed and used according to the NIH guidelines, and this study was approved by the Ethics Committees on Animal Experimentation of Binzhou Medical University (Permit no.

SCXK20160006).

\section{Consent for publication}

Not applicable.

\section{Competing interests}

The authors declare that they have no conflicts of interest.

\section{References}


1. Silvestrini M, Rizzato B, Placidi F, Baruffaldi R, Bianconi A, Diomedi M. Carotid artery wall thickness in patients with obstructive sleep apnea syndrome. Stroke. 2002;33(7):1782-1785.

2. D'Rozario AL, Cross NE, Vakulin A, Bartlett DJ, Wong KKH, Wang D, et al. Quantitative electroencephalogram measures in adult obstructive sleep apnea - Potential biomarkers of neurobehavioural functioning. Sleep Med Rev. 2017;36:29-42.

3. Liu L, MacKenzie KR, Putluri N, Maletić-Savatić M, Bellen HJ. The Glia-Neuron Lactate Shuttle and Elevated ROS Promote Lipid Synthesis in Neurons and Lipid Droplet Accumulation in Glia via APOE/D. Cell Metab. 2017;26(5):719-737.

4. Inloes JM, Kiosses WB, Wang H, Walther TC, Farese RV Jr, Cravatt BF. Functional Contribution of the Spastic Paraplegia-Related Triglyceride Hydrolase DDHD2 to the Formation and Content of Lipid Droplets. Biochemistry. 2018;57(5):827-838.

5. Han X, Zhu J, Zhang X, Song Q, Ding J, Lu M, et al. Plin4-Dependent Lipid Droplets Hamper Neuronal Mitophagy in the MPTP/p-Induced Mouse Model of Parkinson's Disease. Front Neurosci. 2018;12:397.

6. Hamilton LK, Dufresne M, Joppé SE, Petryszyn S, Aumont A, Calon F, et al. Aberrant Lipid Metabolism in the Forebrain Niche Suppresses Adult Neural Stem Cell Proliferation in an Animal Model of Alzheimer's Disease. Cell Stem Cell. 2015;17(4):397-411.

7. Fan J, Shan C, Kang HB, Elf S, Xie J, Tucker M, et al. Tyr phosphorylation of PDP1 toggles recruitment between ACAT1 and SIRT3 to regulate the pyruvate dehydrogenase complex. Mol Cell. 2014;53(4):534-48.

8. Walther TC, Chung J, Farese RV Jr. Lipid Droplet Biogenesis. Annu Rev Cell Dev Biol. 2017;33:491510.

9. Hunkeler M, Hagmann A, Stuttfeld E, Chami M, Guri Y, Stahlberg H, et al. Structural basis for regulation of human acetyl-CoA carboxylase. Nature. 2018;558(7710):470-474.

10. Prabhakar NR, Kumar GK, Peng YJ. Sympatho-adrenal activation by chronic intermittent hypoxia. J Appl Physiol (1985).2012;113:1304-1310.

11. Zhao XJ, Yu HW, Yang YZ, Wu WY, Chen TY, Jia KK, et al. Polydatin prevents fructose-induced liver inflammation and lipid deposition through increasing miR-200a to regulate Keap1/Nrf2 pathway. Redox Biol. 2018;18:124-137.

12. Tian J, Fu F, Li G, Wang Y, Gao Y, Liu Z, et al. SMND-309, a novel derivate of salvianolic acid B, ameliorates cerebral infarction in rats: characterization and role. Brain Res. 2009;1263:114-21.

13. Yang J, Zhang G, Tian J, Li C, Jiang W, Xing Y, et al. Cardioprotective effect of SMND-309, a novel derivate of salvianolic acid $B$ on acute myocardial infarction in rats. Basic Clin Pharmacol Toxicol. 2010;106(4):317-23.

14. Zhu H, Zou L, Tian J, Du G, Gao Y. SMND-309, a novel derivative of salvianolic acid B, protects rat brains ischemia and reperfusion injury by targeting the JAK2/STAT3 pathway. Eur J Pharmacol. 2013;714(1-3):23-31. 
15. Chen J, Guccini I, Di Mitri D, Brina D, Revandkar A, Sarti M, et al. Compartmentalized activities of the pyruvate dehydrogenase complex sustain lipogenesis in prostate cancer. Nat Genet. 2018;50(2):219228.

16. Li W, Yu Y, Li D, Xu N, Fang J, Sun Y, et al. TLR2 deficiency attenuated chronic intermittent hypoxiainduced neurocognitive deficits. Int Immunopharmacol. 2020;81:106284.

17. Dyugovskaya L, Polyakov A, Cohen-Kaplan V, Lavie P, Lavie L. Bax/Mcl-1 balance affects neutrophil survival in intermittent hypoxia and obstructive sleep apnea: effects of p38MAPK and ERK1/2 signaling. J Transl Med. 2012;10:211.

18. Moy SS, Nadler JJ, Perez A, Barbaro RP, Johns JM, Magnuson TR, et al. Sociability and preference for social novelty in five inbred strains: an approach to assess autistic-like behavior in mice. Genes Brain Behav. $2004 ; 3(5): 287-302$.

19. Lee HT, Lee KI, Chen CH, Lee TS. Genetic deletion of soluble epoxide hydrolase delays the progression of Alzheimer's disease. J Neuroinflammation. 2019;16(1):267.

20. Shoji H, Takao K, Hattori S, Miyakawa T. Contextual and cued fear conditioning test using a video analyzing system in mice. J Vis Exp. 2014;1(85):50871.

21. Hu R, Zheng L, Zhang T, Gao G, Cui Y, Cheng Z, et al. Molecular mechanism of hippocampal apoptosis of mice following exposure to titanium dioxide nanoparticles. J Hazard Mater. 2011;191(13):32-40.

22. Ballok DA, Woulfe J, Sur M, Cyr M, Sakic B. Hippocampal damage in mouse and human forms of systemic autoimmune disease. Hippocampus. 2004;14(5):649-61.

23. Liu L, Zhang K, Sandoval H, Yamamoto S, Jaiswal M, Sanz E, et al. Glial lipid droplets and ROS induced by mitochondrial defects promote neurodegeneration. Cell. 2015;160(1-2):177-90.

24. Xu L, Qiu X, Wang S, Wang Q, Zhao XL. NMDA Receptor Antagonist MK801 Protects Against 1Bromopropane-Induced Cognitive Dysfunction. Neurosci. Bull. 2019;35, 347-361.

25. Champagne D, Dupuy JB, Rochford J, Poirier J. Apolipoprotein E knockout mice display procedural deficits in the Morris water maze: analysis of learning strategies in three versions of the task. Neuroscience. 2002;114(3):641-54.

26. Van Dam D, D'Hooge R, Hauben E, Reyniers E, Gantois I, Bakker CE, et al. Spatial learning, contextual fear conditioning and conditioned emotional response in Fmr1 knockout mice. Behav Brain Res. 2000;117(1-2):127-36.

27. Li M, Li X, Lu Y. Obstructive Sleep Apnea Syndrome and Metabolic Diseases. Endocrinology. 2018;159(7):2670-2675.

28. Suh J, Rivest AJ, Nakashiba T, Tominaga T, Tonegawa S. Entorhinal cortex layer III input to the hippocampus is crucial for temporal association memory. Science. 2011;334(6061):1415-20.

29. Lu HD, Liu ZC, Zhou LY, Zhou J, Feng XR, Wang B. Influence of the TLR4-mediated p38MAPK signaling pathway on chronic intermittent hypoxic-induced rat's oxidative stress and inflammatory cytokines in rats. Eur Rev Med Pharmacol Sci. 2019;23(1):352-360. 
30. Ren H, Qiu W, Lu Q, Peng C, Ding Y, Zhu B, et al. Potential contribution of microRNA-125b targeting p38MAPK to relieving intermittent hypoxia-induced dementia of rat models. J Clin Neurosci. 2019;64:234-241.

31. Kiernan EA, Smith SM, Mitchell GS, Watters JJ. Mechanisms of microglial activation in models of inflammation and hypoxia: Implications for chronic intermittent hypoxia. J Physiol. 2016;594(6):1563-77.

32. Rovira-Llopis S, Bañuls C, Diaz-Morales N, Hernandez-Mijares A, Rocha M, Victor VM. Mitochondrial dynamics in type 2 diabetes: Pathophysiological implications. Redox Biol. 2017;11:637-645.

33. Ding W, Cai Y, Wang W, Ji L, Dong Y, Zhang X, et al. Adiponectin protects the kidney against chronic intermittent hypoxia-induced injury through inhibiting endoplasmic reticulum stress. Sleep Breath. 2016;20(3):1069-74.

34. Maxfield FR, Tabas I. Role of cholesterol and lipid organization in disease. Nature. 2005;438(7068):612-21.

35. Dahlman I, Kaaman M, Jiao H, Kere J, Laakso M, Arner P. The CIDEA gene V115F polymorphism is associated with obesity in Swedish subjects. Diabetes. 2005;54(10):3032-4.

36. Carr RM, Peralta G, Yin X, Ahima RS. Absence of perilipin 2 prevents hepatic steatosis, glucose intolerance and ceramide accumulation in alcohol-fed mice. PLoS One. 2014;9(5):e97118.

37. Ruipérez V, Darios F. Alpha-synuclein, lipids and Parkinson's disease. Prog. Lipid Res. 2010; 49:420428.

38. Klemm RW, Norton JP, Cole RA, Li CS, Park SH, Crane MM, et al. A conserved role for atlastin GTPases in regulating lipid droplet size. Cell Rep. 2013;3(5):1465-75.

39. Konige M, Wang H, Sztalryd C. Role of adipose specific lipid droplet proteins in maintaining whole body energy homeostasis. Biochim Biophys Acta. 2014;1842(3):393-401.

40. Schmitt F, Hussain G, Dupuis L, Loeffler JP, Henriques A. A plural role for lipids in motor neuron diseases: energy, signaling and structure. Front Cell Neurosci. 2014;8:25.

41. Moldavski O, Amen T, Levin-Zaidman S, Eisenstein M, Rogachev I, Brandis A, et al. Lipid Droplets Are Essential for Efficient Clearance of Cytosolic Inclusion Bodies. Dev Cell. 2015;33(5):603-10.

42. Welte MA, Gould AP. Lipid droplet functions beyond energy storage. Biochim Biophys Acta Mol Cell Biol Lipids. 2017;1862(10 Pt B):1260-1272.

43. Shan C, Kang HB, Elf S, Xie J, Gu TL, Aguiar M, et al. Tyr-94 phosphorylation inhibits pyruvate dehydrogenase phosphatase 1 and promotes tumor growth. J Biol Chem. 2014;289(31):21413-22.

44. Zhong Y, Li X, Ji Y, Li X, Li Y, Yu D, et al. Pyruvate dehydrogenase expression is negatively associated with cell stemness and worse clinical outcome in prostate cancers. Oncotarget. 2017;8(8):1334413356.

45. Kuerschner L, Moessinger C, Thiele C. Imaging of lipid biosynthesis: how a neutral lipid enters lipid droplets. Traffic. 2008;9(3):338-52. 
46. Zhang M, Zhao Y, Li Z, Wang C. Pyruvate dehydrogenase kinase 4 mediates lipogenesis and contributes to the pathogenesis of nonalcoholic steatohepatitis. Biochem Biophys Res Commun. 2018;495(1):582-586.

47. Pignataro D, Francia S, Zanetta F, Brenna G, Brandini S, Olivieri A, et al. A missense MT-ND5 mutation in differentiated Parkinson Disease cytoplasmic hybrid induces ROS-dependent DNA Damage Response amplified by DROSHA. Sci Rep. 2017;7(1):9528.

48. Fransen M, Nordgren M, Wang B, Apanasets 0 . Role of peroxisomes in ROS/RNS-metabolism: implications for human disease. Biochim Biophys Acta. 2012;1822(9):1363-73.

49. Pan JH, Lim Y, Kim JH, Heo W, Lee KY, Shin HJ, et al. Root bark of Ulmus davidiana var japonica restrains acute alcohol-induced hepatic steatosis onset in mice by inhibiting ROS accumulation. PLoS One. 2017;12(11):e0188381.

50. Nogalska A, Sucajtys-Szulc E, Swierczynski J. Leptin decreases lipogenic enzyme gene expression through modification of SREBP-1c gene expression in white adipose tissue of aging rats. Metabolism. 2005;54(8):1041-7.

51. Su CY, Ming QL, Rahman K, Han T, Qin LP. Salvia miltiorrhiza: Traditional medicinal uses, chemistry, and pharmacology. Chin J Nat Med. 2015;13(3):163-82.

52. Wang Y, Zhang J, Han M, Liu B, Gao Y, Ma P, et al. SMND-309 promotes neuron survival through the activation of the PI3K/Akt/CREB-signalling pathway. Pharm Biol. 2016;54(10):1982-90.

\section{Figures}

A

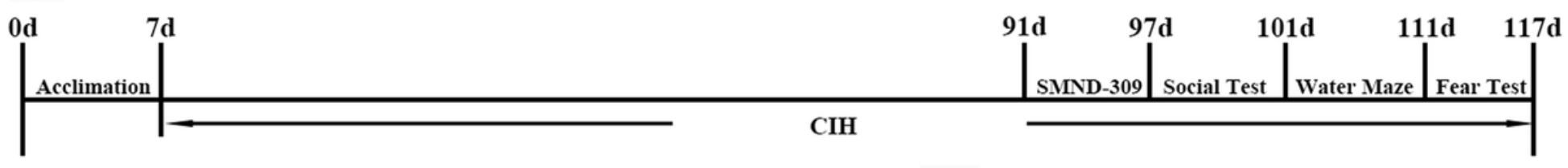

B

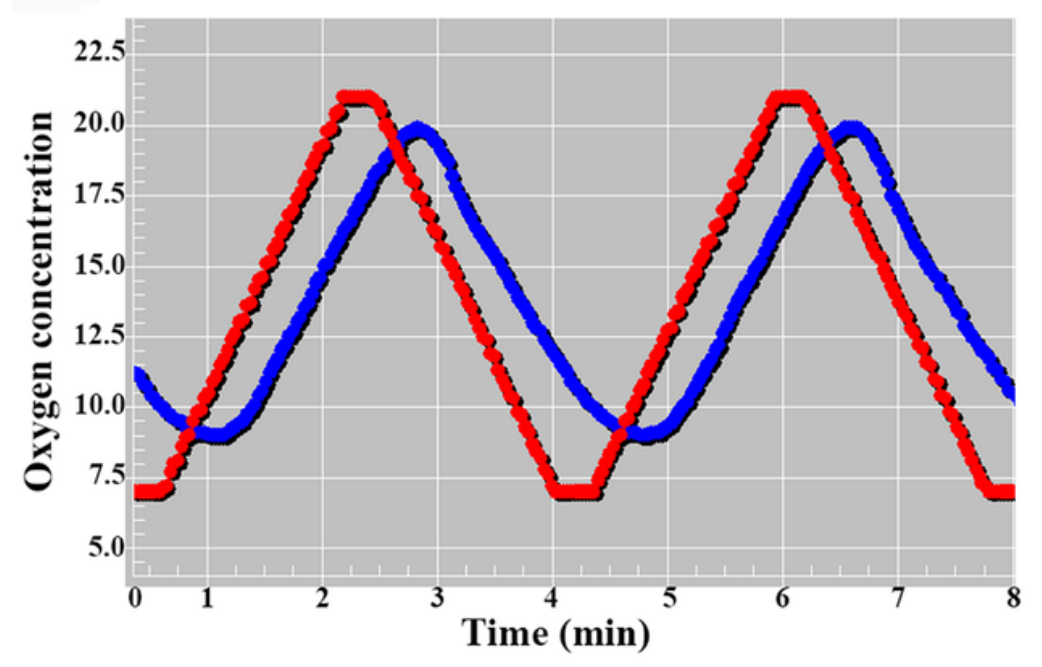

C

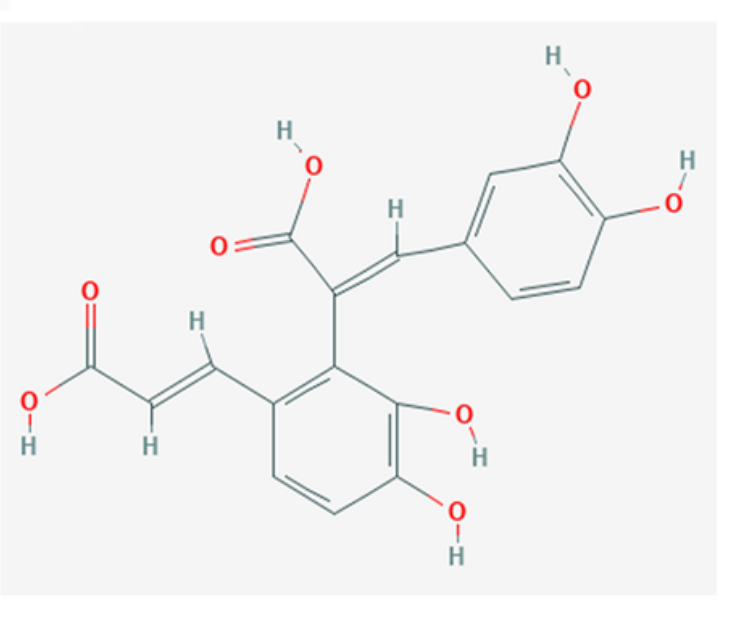

SMND-309

Figure 1 
The process of research and the structure of SMND-309. (A) The study workflow. After 7 days of acclimation, the mice were exposed to the $\mathrm{CIH}$ condition for 12 weeks, and they were treated with SMND309 at the last week. Behavioral tests were conducted as soon as $\mathrm{ClH}$ exposure finished. (B) $\mathrm{The} \mathrm{ClH}$ treatment program. The red line represents the set point. The blue line represents the actual oxygen level. (C) The structure of SMND-309.
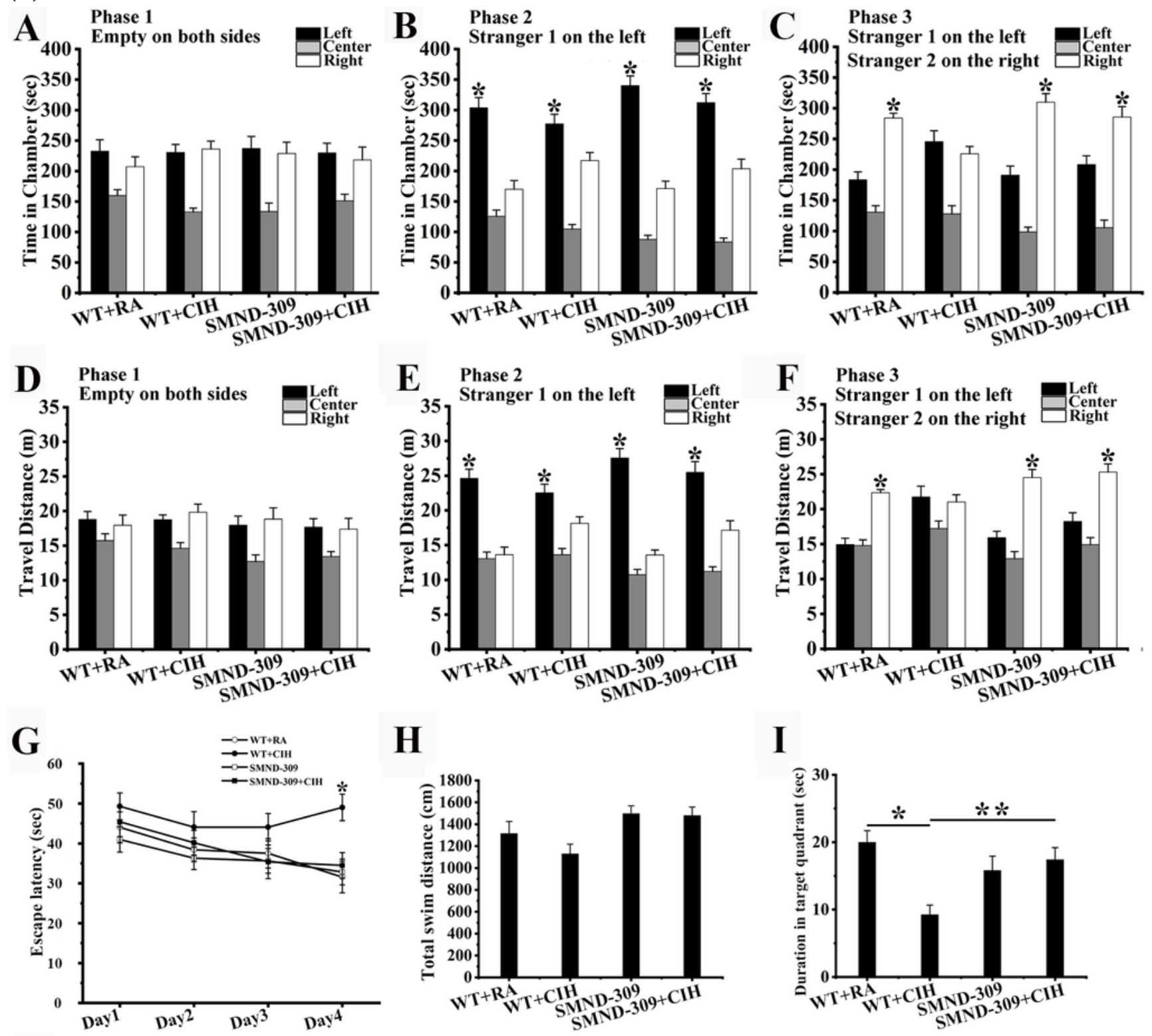

$\mathbf{H}$

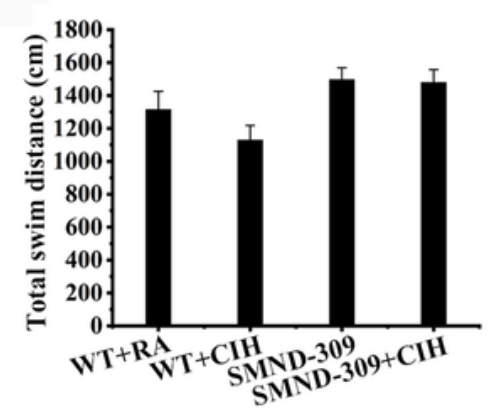

I
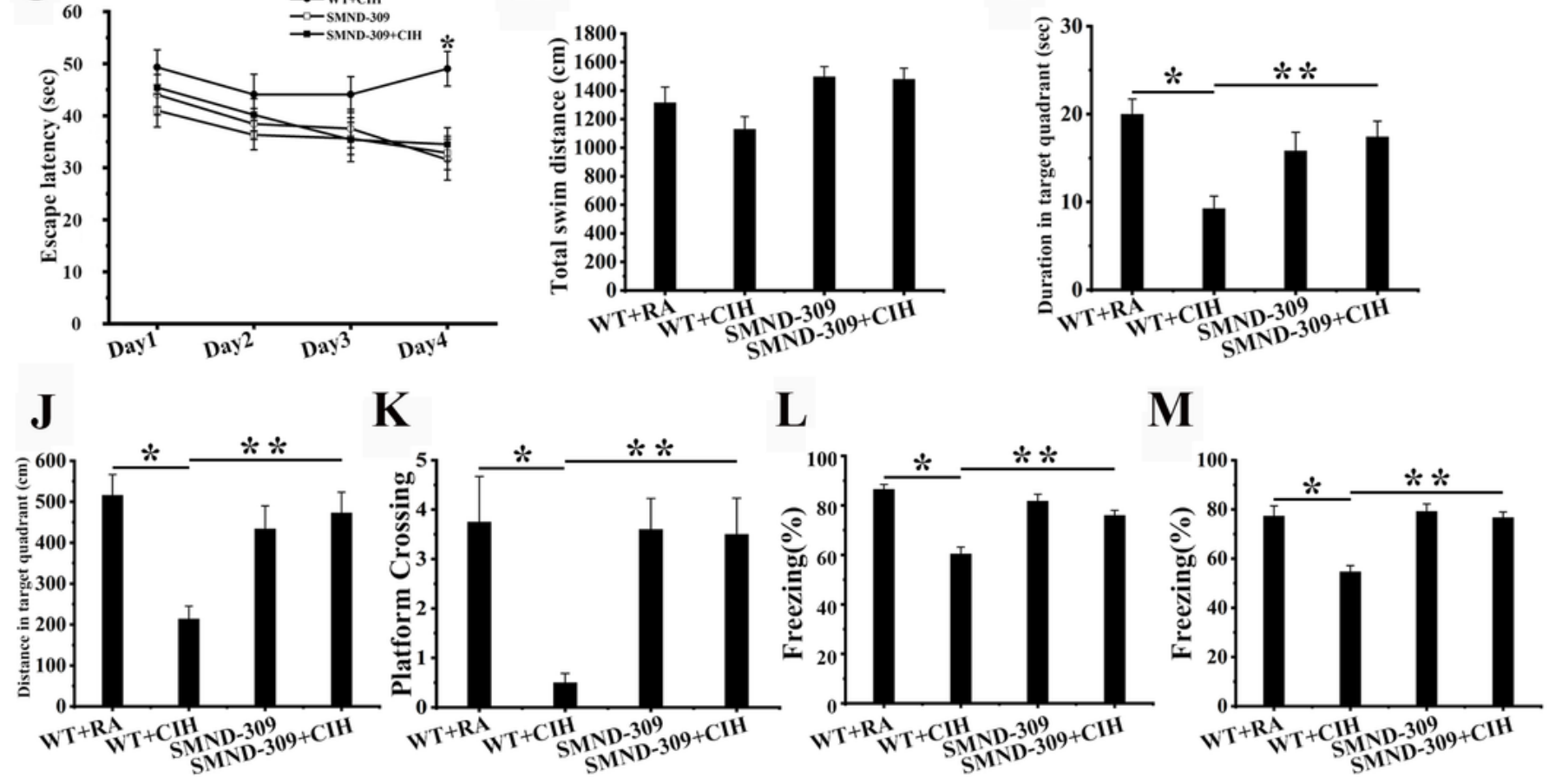

M

Figure 2 
The behavioral performance in mice. (A, D) During phase 1, there was no significant difference between the two sides in all groups. (B, E) During phase 2, all of the mice preferred to stay with the stranger mouse. (C, F) During phase 3, WT+RA, SMND-309 and SMND-309+CIH mice preferred to stay with Stranger 2 than with Stranger $1(p<0.05)$. However, WT+CIH mice spent a similar amount of time with each of the two stranger mice ( $p>0.05)$. (G) The mean escape latencies of the four groups. $(H)$ The total swimming distances among all groups were similar $(p>0.05)$. (I) The time the $W T+C l H$ mice spent in the target quadrant was much shorter than the time the WT+RA mice spent $(p<0.05)$, and this was improved by SMND-309 treatment $(p<0.05)$. (J) WT+RA mice traveled longer distances in the target quadrant than the WT+ClH mice $(p<0.05)$, and after SMND-309 treatment, the damage was improved $(p<0.05)$. (K) The number of platform crossings of WT+ClH mice was less than WT+RA mice $(\mathrm{p}<0.05)$, but it was improved by treating with SMND-309 $(\mathrm{p}<0.05)$. $(\mathrm{L}, \mathrm{M})$ The fear memory was damaged by $\mathrm{ClH}$ exposure, and SMND309 alleviated the damage. ${ }^{*} p<0.05$ Stranger 1 vs. empty cage. ${ }^{*} p<0.05$ Stranger 1 vs. Stranger $2 .{ }^{*} p<0.05$ WT group vs. WT ClH group. ${ }^{\star \star} \mathrm{p}<0.05 \mathrm{WT} \mathrm{ClH}$ group vs. SMND-309 $\mathrm{ClH}$ group. $\mathrm{N}=10$ for each group. Data are shown as the mean \pm SEM. The results of social tests were analyzed by one-way ANOVA and a Bonferroni test. The results of escape latency were checked by three-way mixed ANOVA. Others were measured by two-way ANOVA.
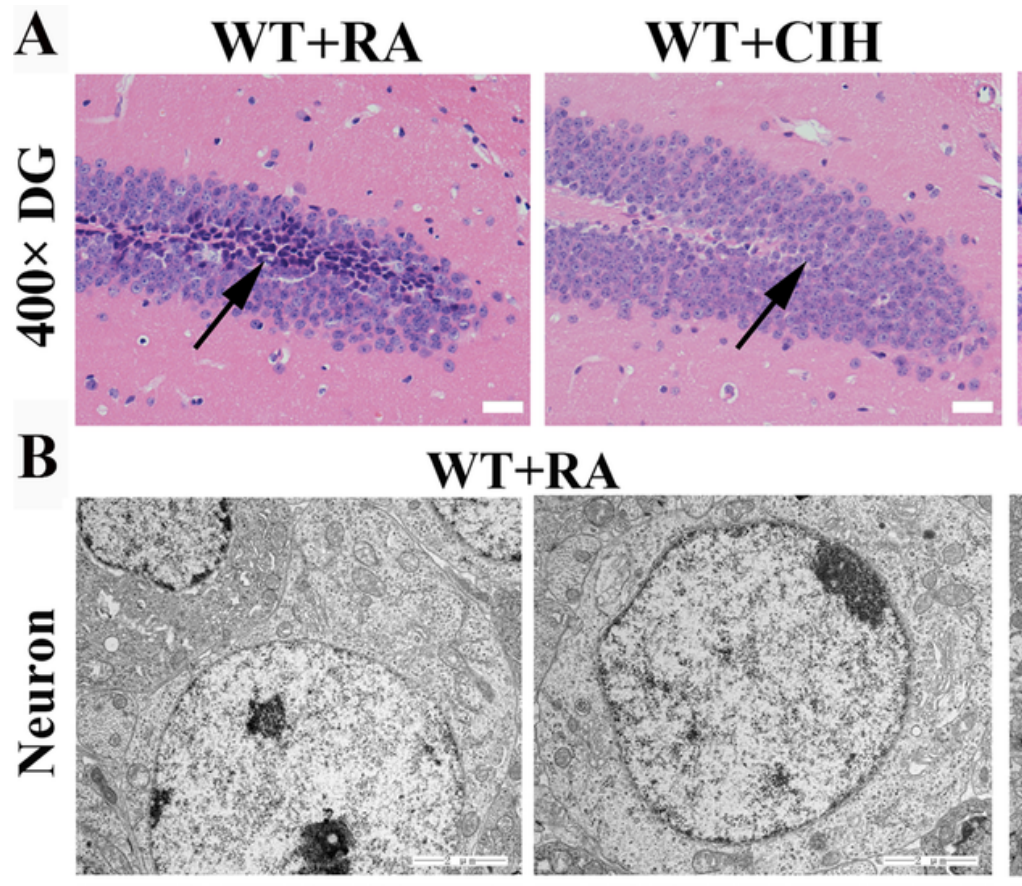

SMND-309

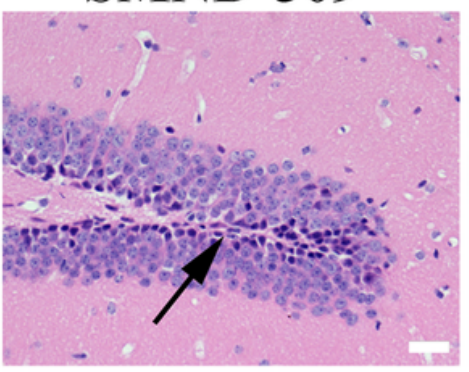

SMND-309+CIH
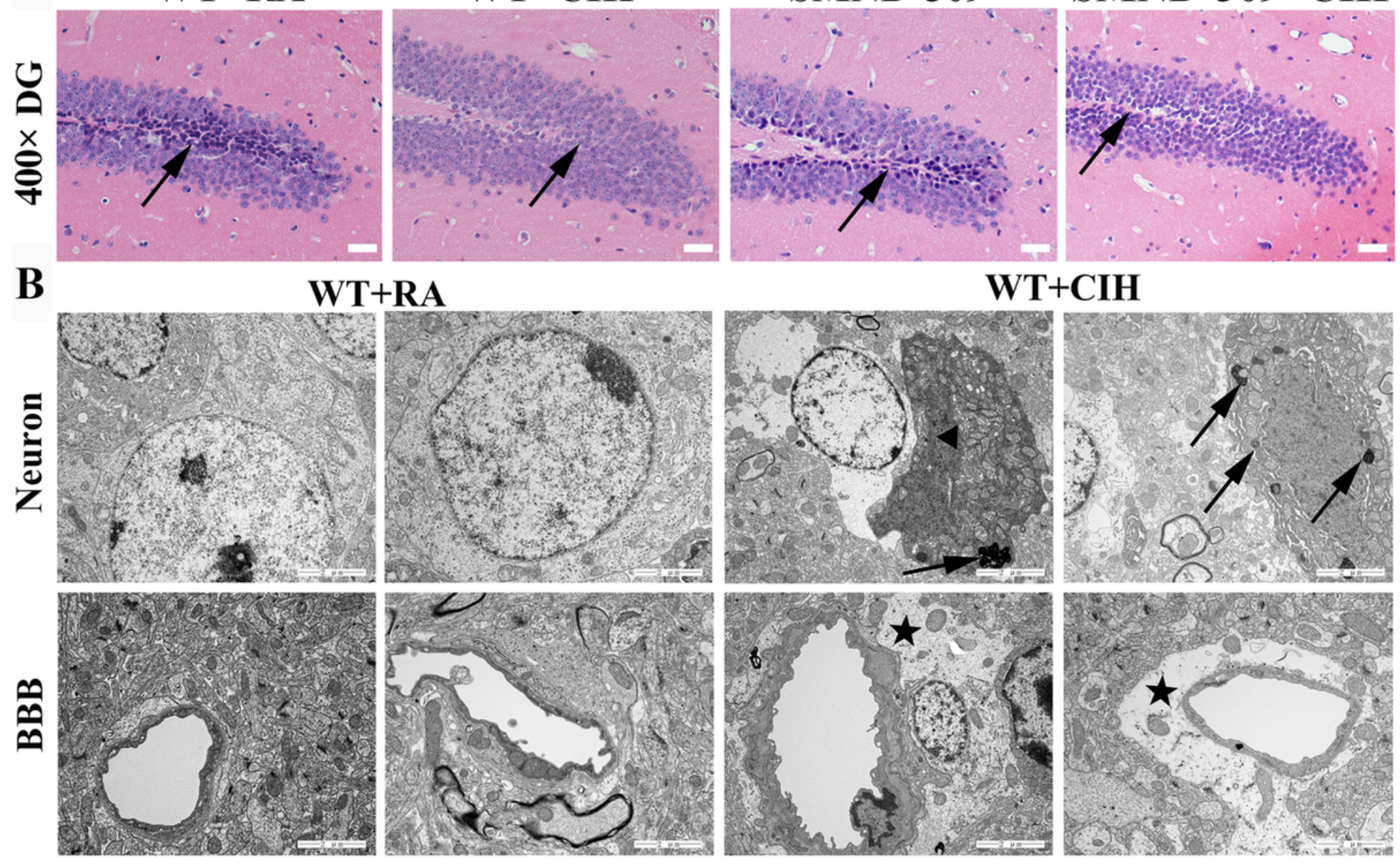

WT+CIH
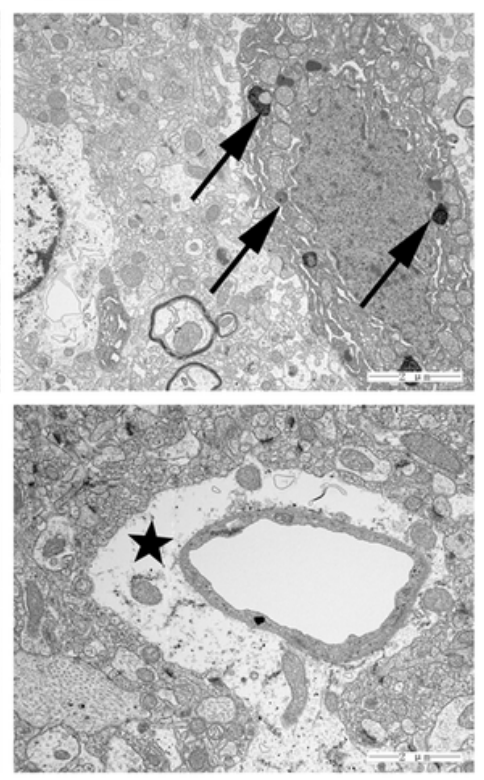

Figure 3 
Pathological changes of DG area. (A) H\&E staining of the hippocampus. In the SGZ of the WT+CIH group, NBs were hardly observed (black arrow), but SMND-309 treatment relieved the damage. Original magnification: 400x. (B) The TEM results of the hippocampus. Neurons of the WT+ClH group exhibited cytoplasmic vacuolization, LDs accumulation (black arrow) and mitochondrial disruption (black triangle). Moreover, glial cells of the $\mathrm{WT}+\mathrm{ClH}$ group presented edema (black star), especially in the area surrounding the BBB. Original magnification: $15000 \times$. All experiments were repeated three times.

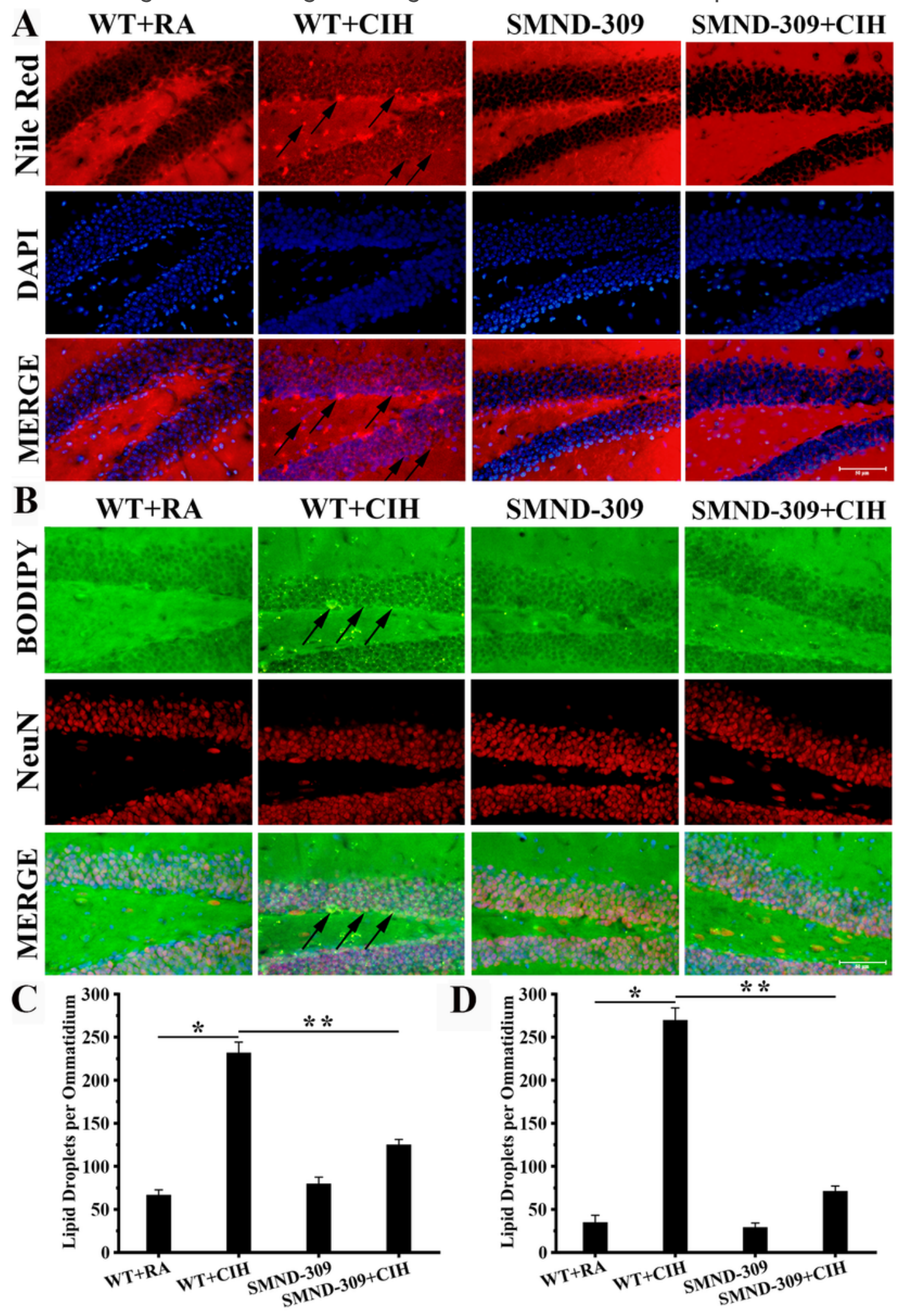

Figure 4 
LDs accumulation in neuron. To detect LDs accumulation in the hippocampal neurons, Nile red (red) and DAPI (blue), BODIPY (483/503) (green) and NeuN (red) were used. (A) LDs accumulation could be detected (red, black arrow) in the WT+ClH mice, and treated with SMND-309 decreased this change. (B) Abundant BODIPY (483/503) fluorescence could be detected in the neurons of the DG area under $\mathrm{CIH}$ condition, but the fluorescence became dim after SMND-309 treatment. (C) Quantification of LDs accumulation in neurocytes. (D) Quantification of LDs accumulation in neurons. ${ }^{*} p<0.05$ WT group vs. WT $\mathrm{ClH}$ group. ${ }^{* \star} \mathrm{p}<0.05 \mathrm{WT} \mathrm{ClH}$ group vs. SMND-309 ClH group. All experiments were repeated three times. Data are shown as the mean \pm SEM. Statistical analysis was tested by two-way ANOVA. Original magnification: $400 x$. 


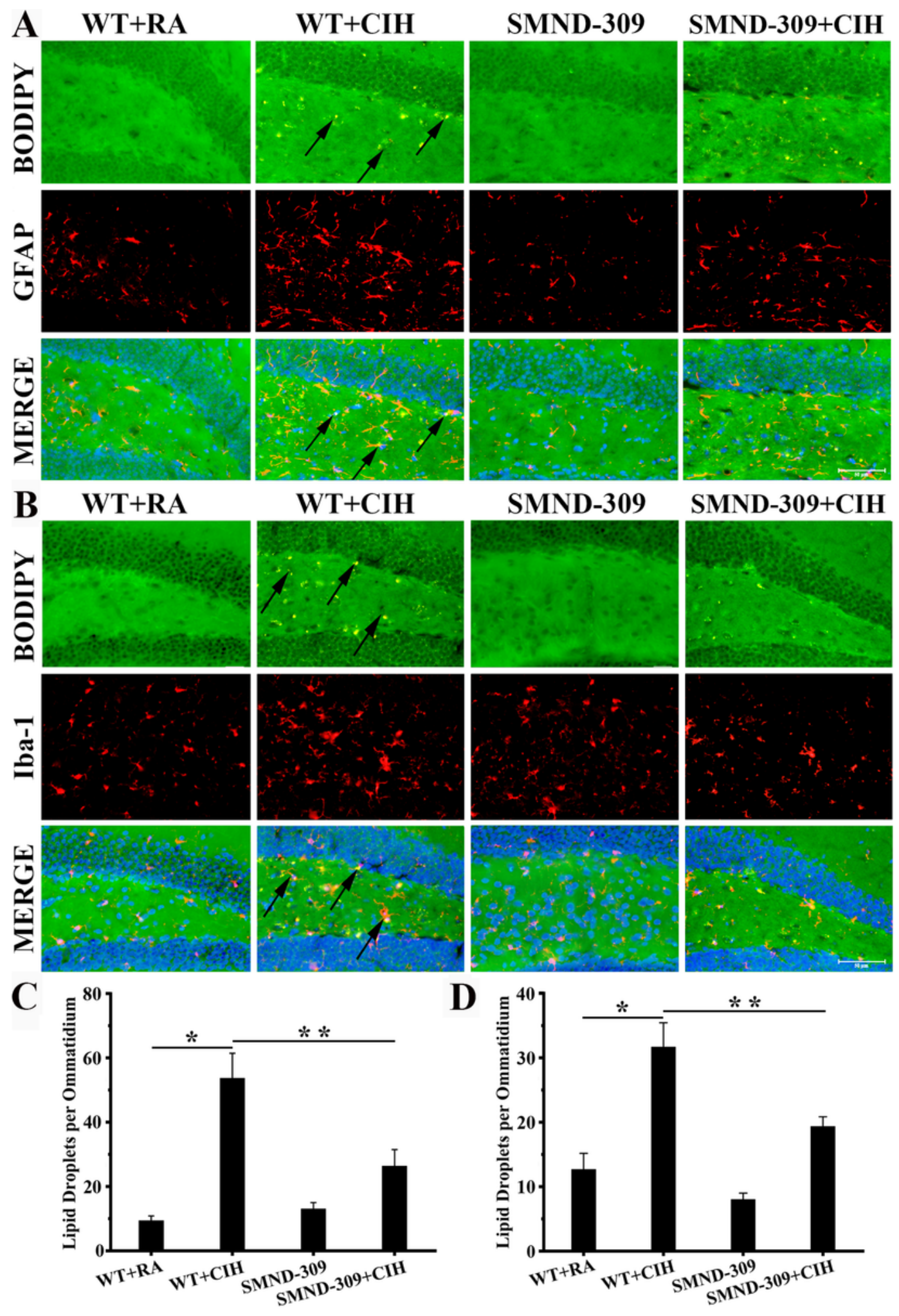

Figure 5

LDs accumulation in glial cells. The microglia marker lba-1 (red), astrocyte marker GFAP (red) and DAPI (blue) were stained with BODIPY (483/503) (green) to observe LDs accumulation in glia. The swollen cell body and increased synapses along with the mass of BODIPY $(483 / 503)$ spots could be observed in glial cells after $\mathrm{ClH}$ exposure. With SMND-309 treatment, glial injuries were relieved. LDs accumulated (yellow, black arrow) could also be detected in astrocytes (A) and microglia (B). (C) Quantification of LDs 
accumulation in astrocytes. (D) Quantification of LDs accumulation in microglia. * $p<0.05$ WT group vs. WT ClH group. ${ }^{* *} \mathrm{p}<0.05 \mathrm{WT} \mathrm{ClH}$ group vs. SMND-309 ClH group. All experiments were repeated three times. Data are shown as the mean \pm SEM. Statistical analysis included two-way ANOVA. Original magnification: $400 x$.

A WT+RA
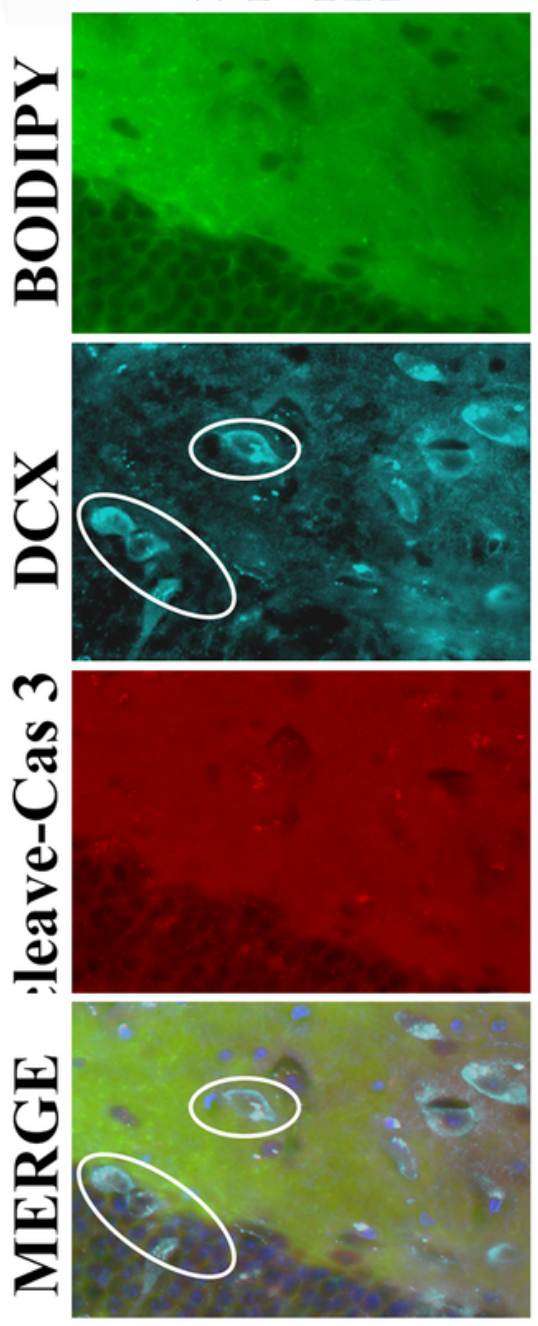

B

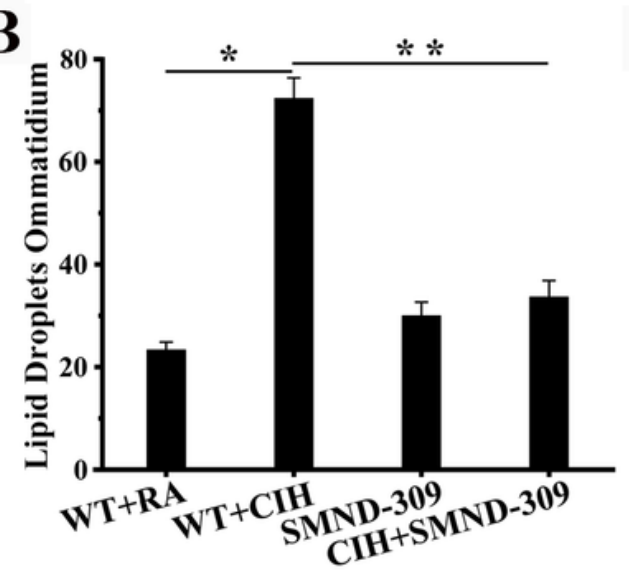

WT+CIH
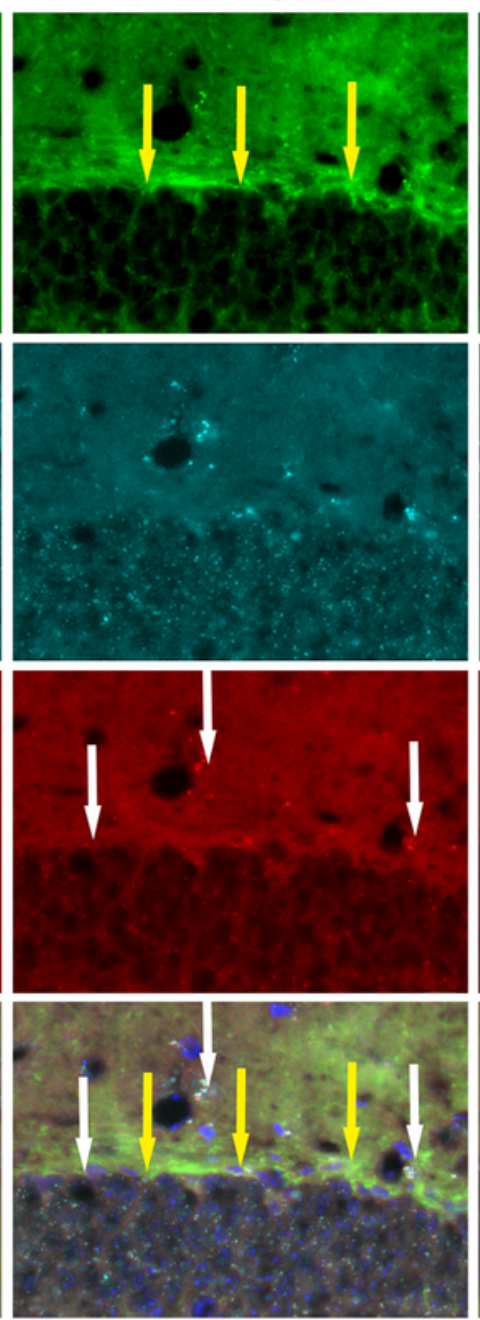

C

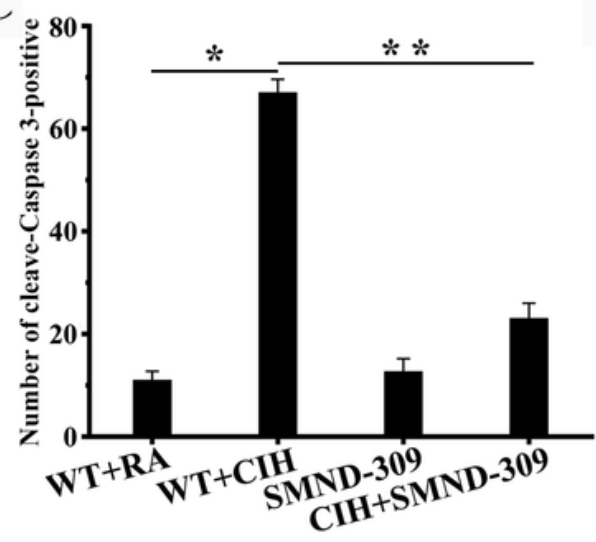

SMND-309
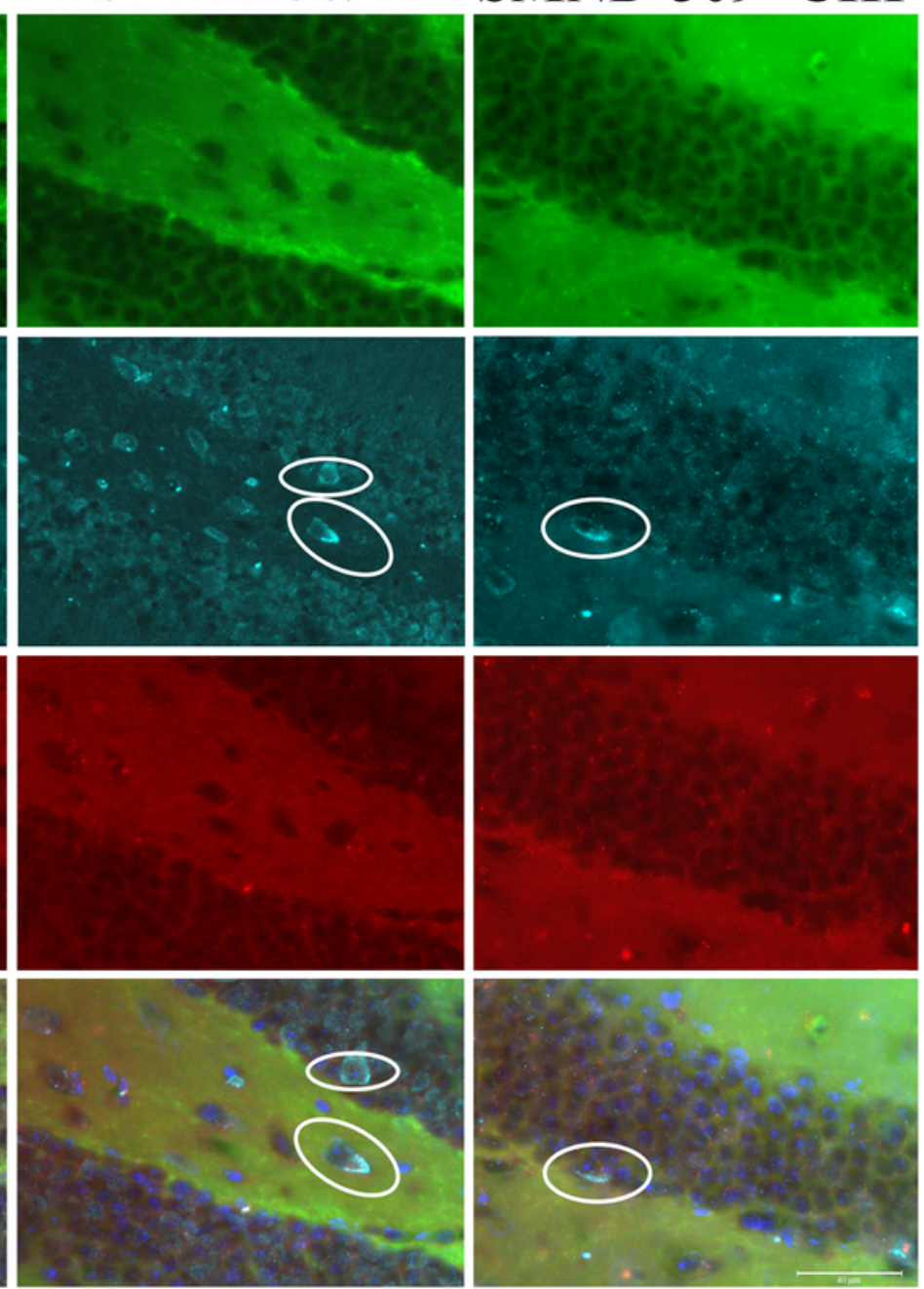

D

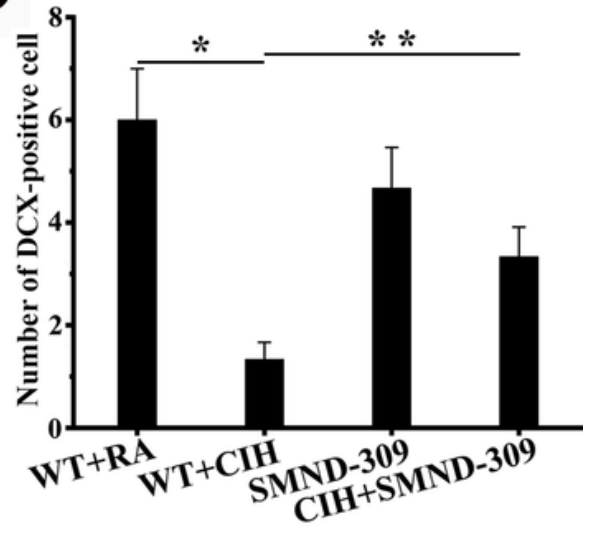

Figure 6 
LDs accumulation-induced NBs apoptosis. Fluorescent immunocytochemistry staining was as follows: NB marker DCX (sky blue), cleaved caspase 3 (red), BODIPY (483/503) (green) and DAPI (blue). (A) Seldom DCX-positive cells (blue, white circle) in the SGZ of WT+CIH mice could be observed. Moreover, a larger number of BODIPY (483/503) (green, yellow arrow) and cleaved caspase 3-positive cells (red, white arrow) merged. SMND-309 treatment ameliorated these injuries. (B) Quantification of LDs accumulation. (C) Quantification of cleaved caspase 3-positive cell. (D) Quantification of DCX-positive cell. ${ }^{*}<0.05$ WT group vs. WT CIH group. ${ }^{*} \mathrm{p}<0.05$ WT $\mathrm{ClH}$ group vs. SMND-309 CIH group. All experiments were repeated three times. Data are shown as the mean \pm SEM. Statistical analysis was performed with two-way ANOVA. Original magnification: 600x. 

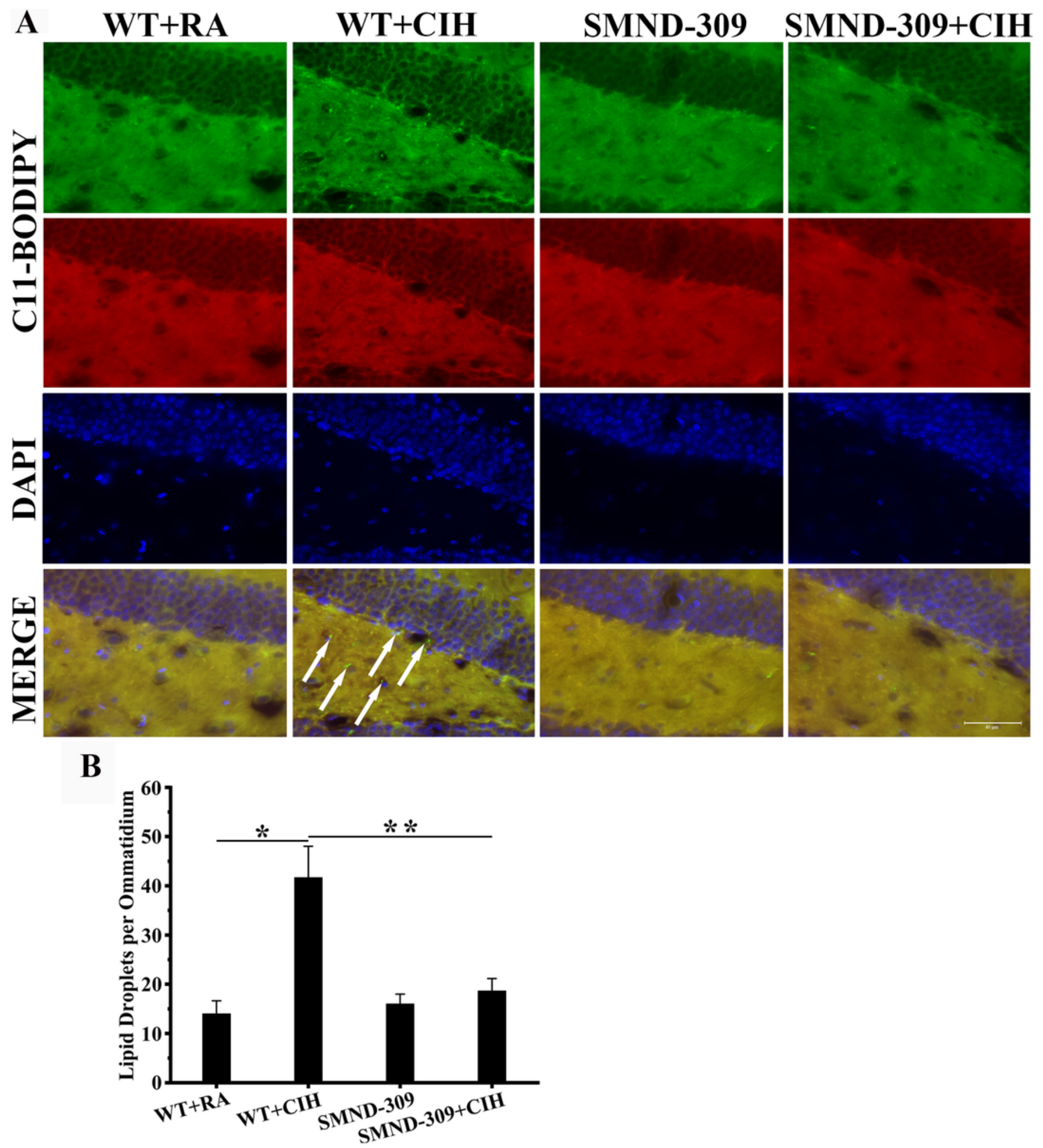

Figure 7

Affecting the pathway of PDP1/PDHA1 did not improve the damage caused by $\mathrm{ClH}$ exposure (A) The study workflow. After 7 days of acclimation, the mice were exposed to the $\mathrm{ClH}$ condition for 12 weeks, and they were treated with 3-FP at the last 3 weeks. Fear test were conducted as soon as $\mathrm{ClH}$ exposure finished. (B) The structure of 3-FP. (C) The freezing level of contextual memory among 4 groups. (D) The freezing level of cued memory among 4 groups. (E) H\&E stained of the hippocampus. Each experiment 
was repeated three times. Data are shown as the mean \pm SEM. Statistical analysis was performed with two-way ANOVA. * ${ }^{*}<0.05$ WT group vs WT CIH group. Magnification: $400 \times$. The local details are enlarged in the rectangular frame.
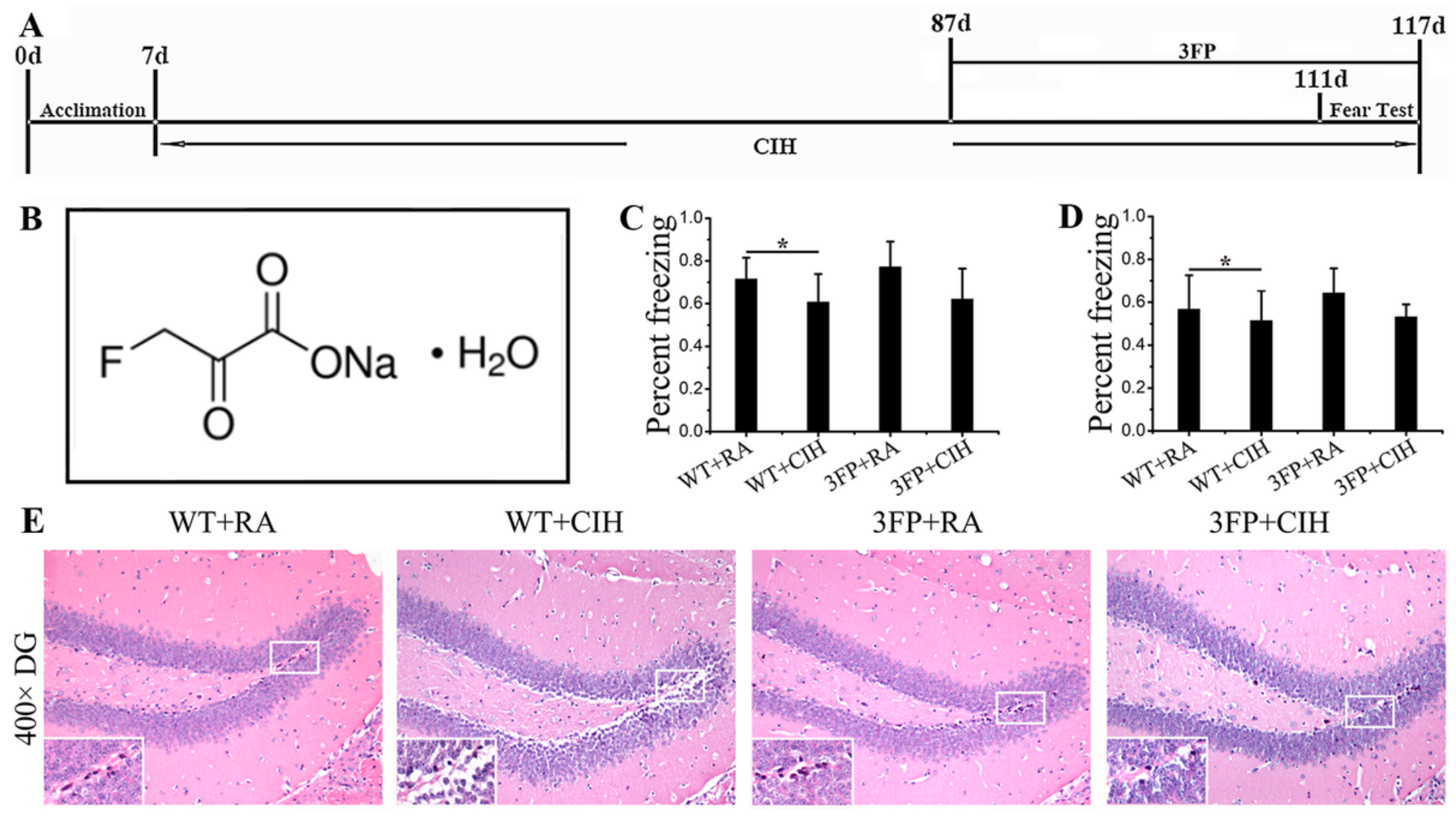

$3 F P+R A$

$3 \mathrm{FP}+\mathrm{CIH}$

Figure 8

$\mathrm{CIH}$ treatment did not significant influence the expression of PDP1/PDHA1 in hippocampus. (A) Nile Red staining of hippocampus. (Scale bar: $20 \mu \mathrm{m}$ ) (B) Quantification of LDs accumulation. (C, D) PDP1 and PDHA1 expression patterns were determined by RT-PCR. (E-H) Western blot assays were performed to determine the expression level of PDP1/PDHA1, and the gray value of the band was measured. (I) The PDC activity of hippocampus. $(J)$ Total levels of acetyl-CoA in hippocampus. Each experiment was repeated three times. Data are shown as the mean \pm SEM. Statistical analysis was performed with twoway ANOVA. ${ }^{*} p<0.05$ WT group vs WT CIH group. Magnification: 400x. The local details are enlarged in the rectangular frame. 

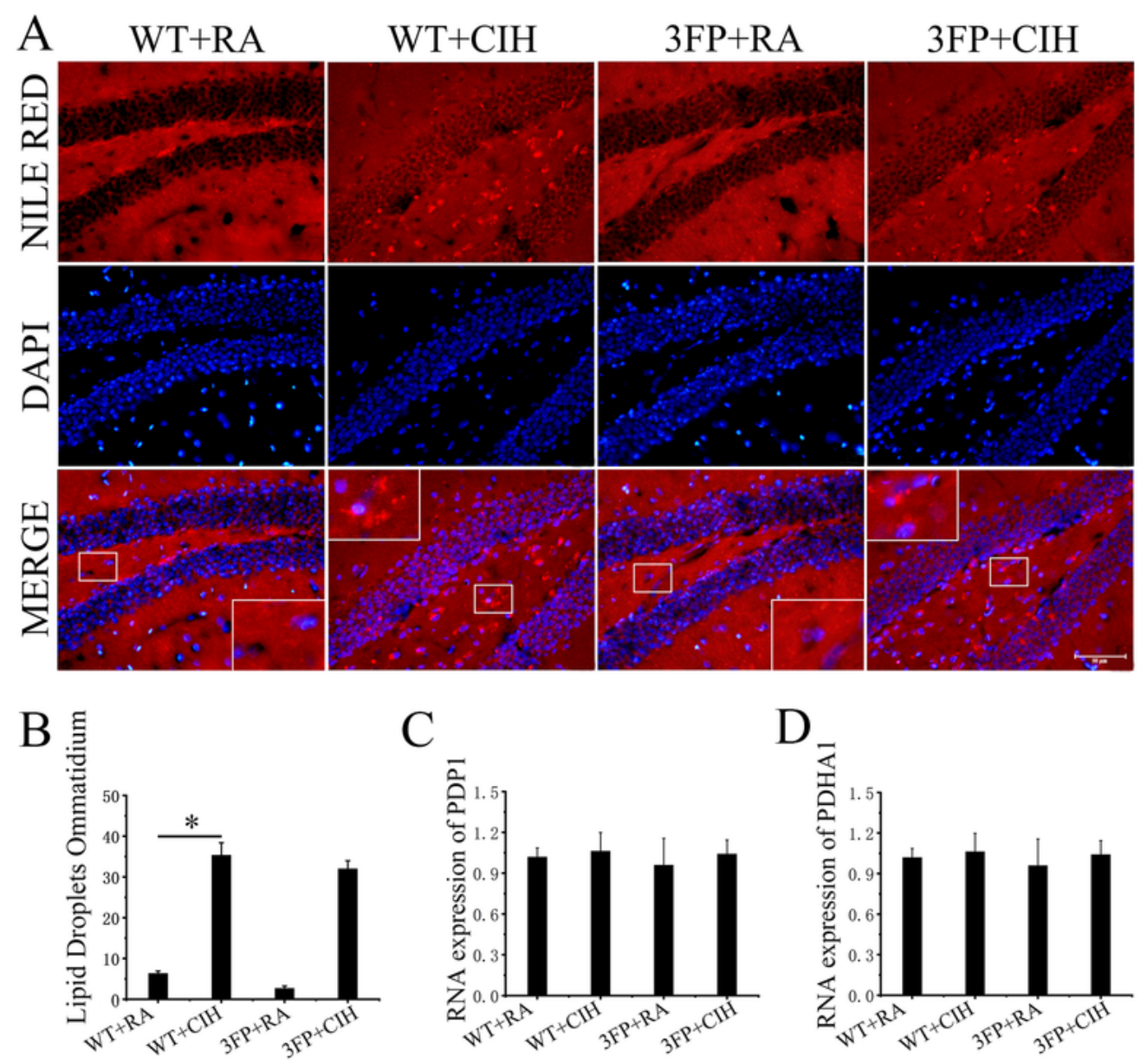

$\mathrm{C}$
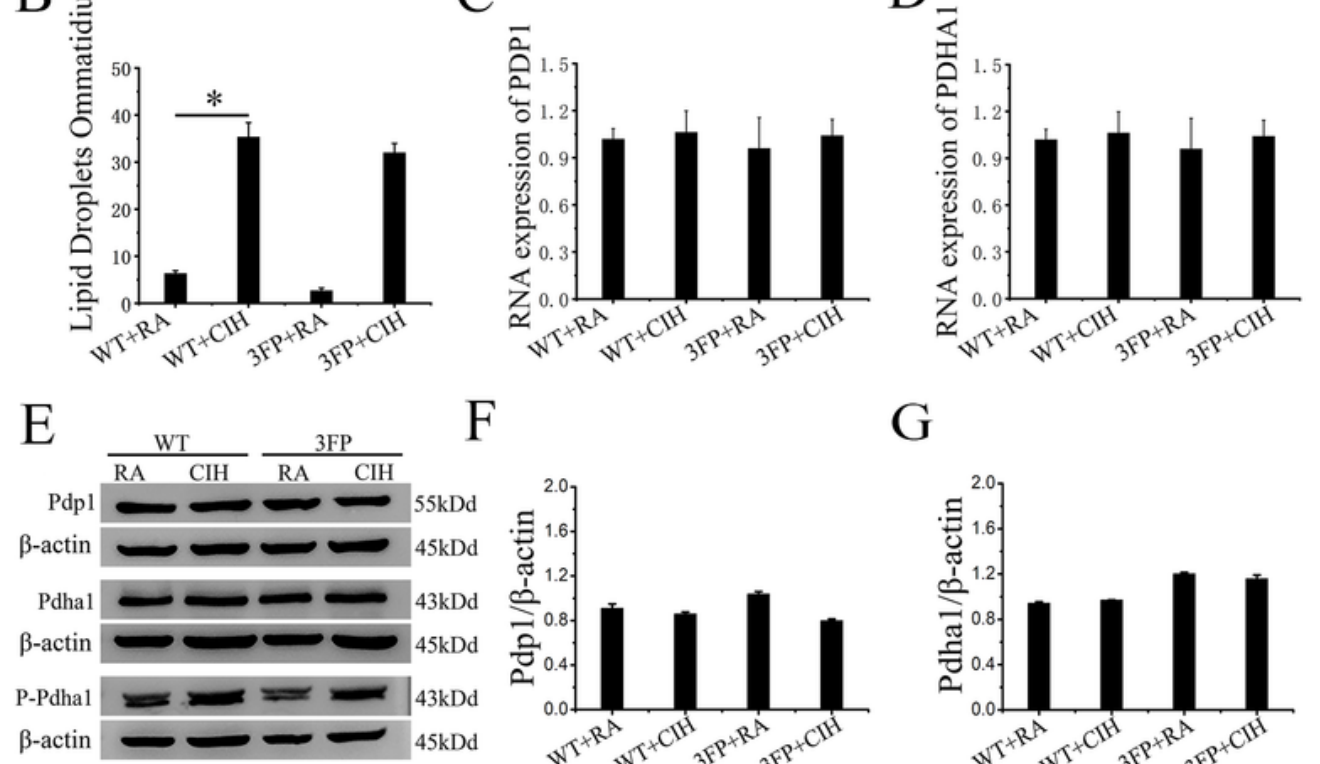

$\mathrm{F}$

G
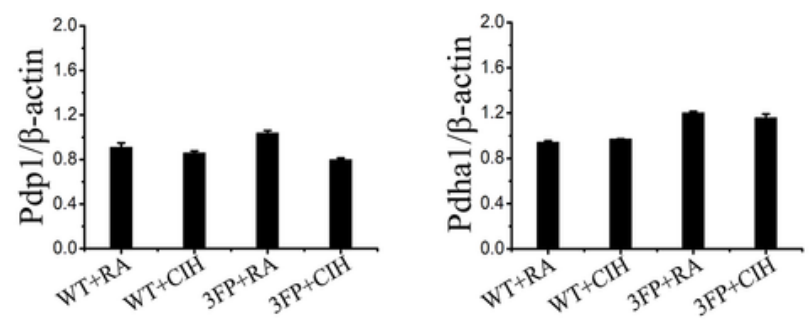

$\mathrm{H}$
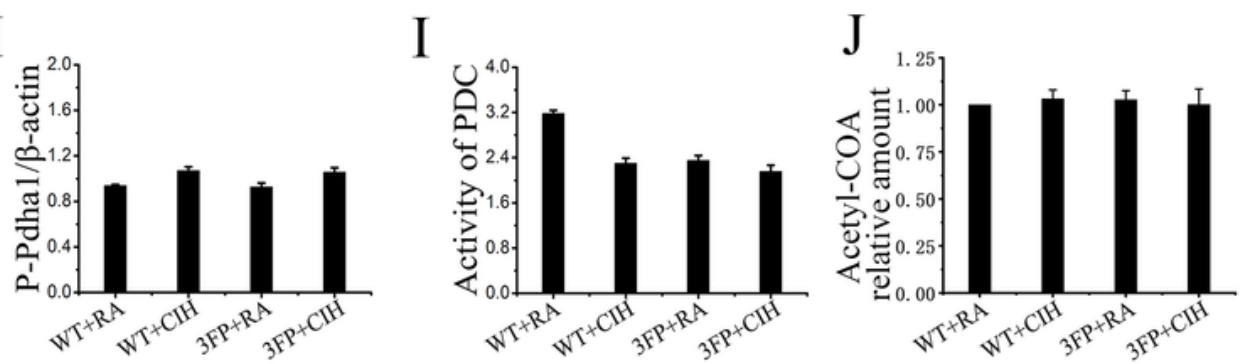

Figure 9

ROS level and JNK/SREBP/ACC expression. (A-G) The ROS level was highly enhanced in the hippocampus of WT+CIH exposure $(p<0.05)$, and the JNK/SREBP/ACC pathway was activated. However, these changes were attenuated by SMND-309 treatment $(p<0.05) .{ }^{*} p<0.05$ WT group vs. WT CIH group. ${ }^{\star *} \mathrm{p}<0.05 \mathrm{WT} \mathrm{ClH}$ group vs. SMND-309 ClH group. All experiments were repeated three times. Data are expressed as the mean \pm SEM. Statistical analysis was performed through two-way ANOVA. 

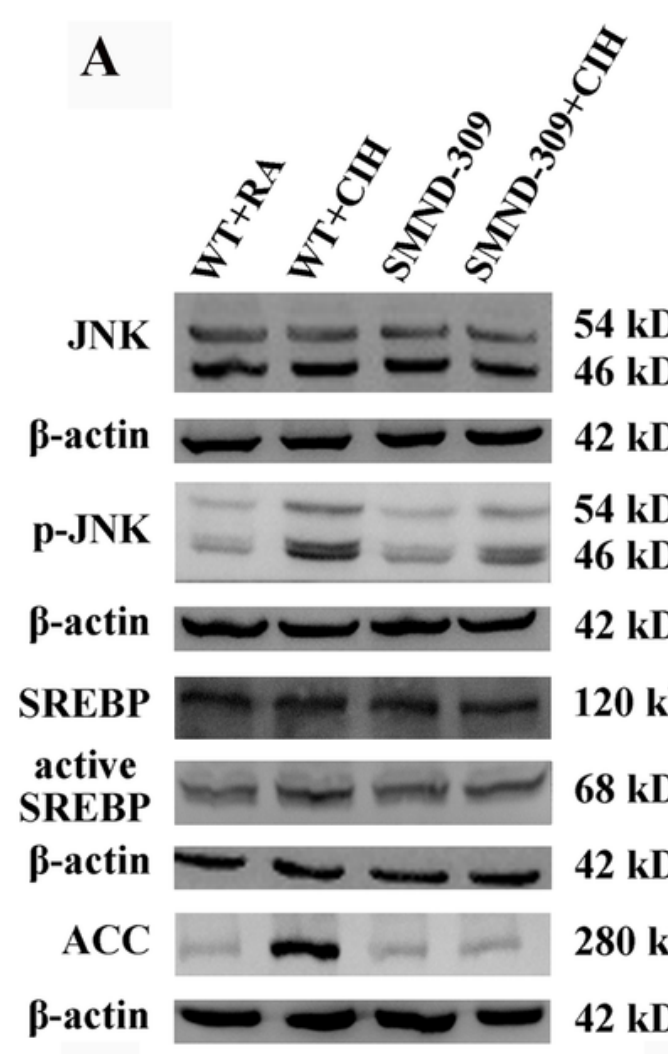

$54 \mathrm{kDd}$
$46 \mathrm{kDd}$

$42 \mathrm{kDd}$

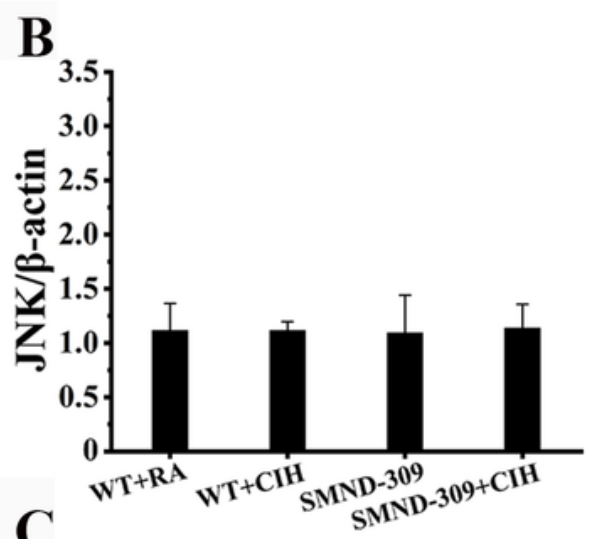

$46 \mathrm{kDd} \quad \mathrm{C}$

42 kDd

120 kDd
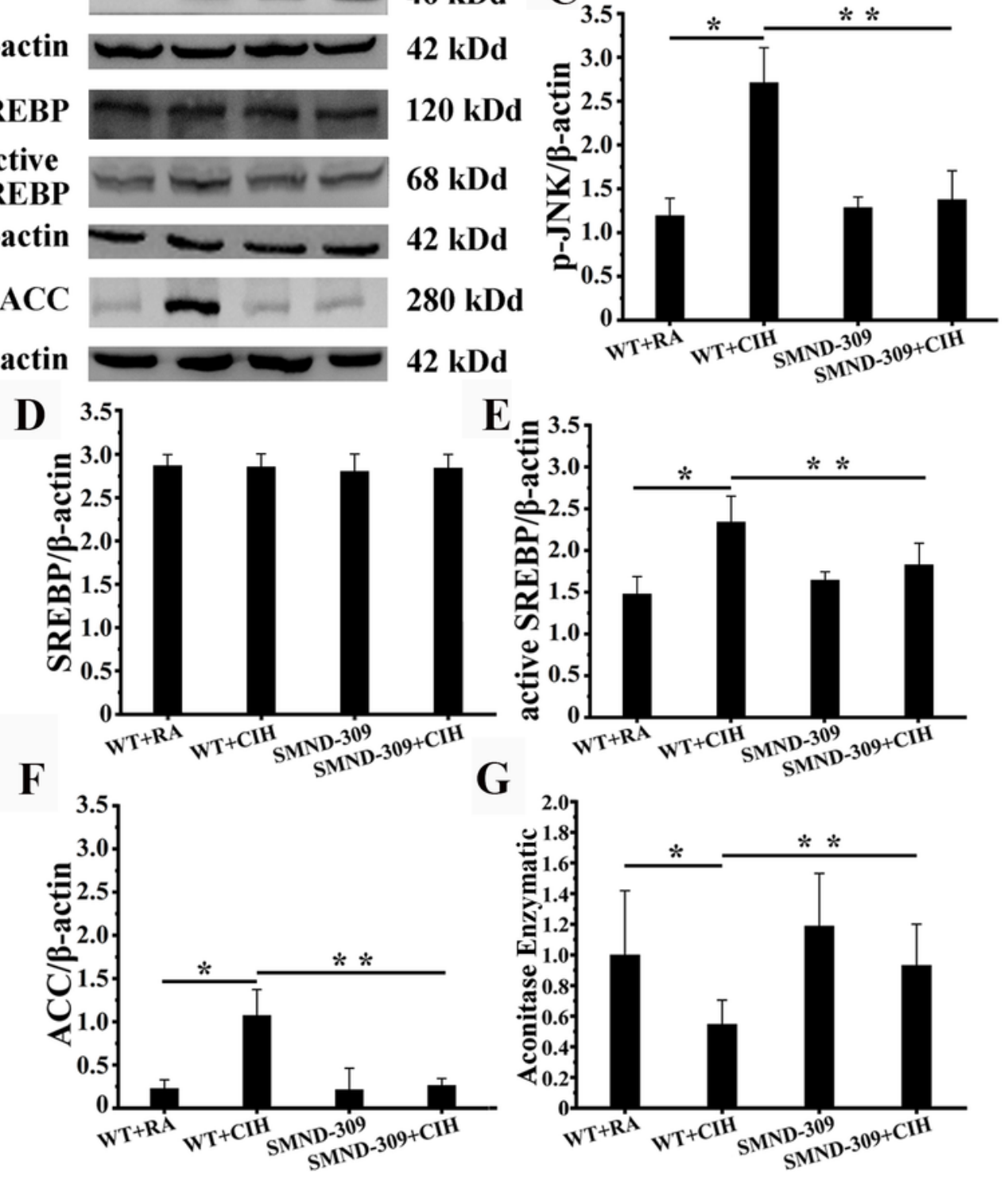

Figure 10

lipid peroxidation in neurocytes. (A) C11-BODIPY (581/591) (red, green) and DAPI (blue) staining of the DG region. Lipid peroxidation could be observed (green, white arrow) in neurocytes and SMND-309 inhibited peroxidation. (B) Quantification of peroxidized lipid in the neurocytes. ${ }^{*} p<0.05$ WT group vs. WT $\mathrm{ClH}$ group. ${ }^{\star \star} \mathrm{p}<0.05 \mathrm{WT} \mathrm{ClH}$ group vs. SMND-309 ClH group. All experiments were repeated three times. 
Data are shown as the mean \pm SEM. Statistical analysis was performed with two-way ANOVA. Original magnification: $400 x$.

\section{Supplementary Files}

This is a list of supplementary files associated with this preprint. Click to download.

- Supplement.docx 